\title{
Nonuniform Sampling of Periodic Bandlimited Signals
}

\author{
Evgeny Margolis, Student Member, IEEE, and Yonina C. Eldar, Senior Member, IEEE
}

\begin{abstract}
Digital processing techniques are based on representing a continuous-time signal by a discrete set of samples. This paper treats the problem of reconstructing a periodic bandlimited signal from a finite number of its nonuniform samples. In practical applications, only a finite number of values are given. Extending the samples periodically across the boundaries, and assuming that the underlying continuous time signal is bandlimited, provides a simple way to deal with reconstruction from finitely many samples. Two algorithms for reconstructing a periodic bandlimited signal from an even and an odd number of nonuniform samples are developed. In the first, the reconstruction functions constitute a basis while in the second, they form a frame so that there are more samples than needed for perfect reconstruction. The advantages and disadvantages of each method are analyzed. Specifically, it is shown that the first algorithm provides consistent reconstruction of the signal while the second is shown to be more stable in noisy environments. Next, we use the theory of finite dimensional frames to characterize the stability of our algorithms. We then consider two special distributions of sampling points: uniform and recurrent nonuniform, and show that for these cases, the reconstruction formulas as well as the stability analysis are simplified significantly. The advantage of our methods over conventional approaches is demonstrated by numerical experiments.
\end{abstract}

Index Terms-Interpolation, nonuniform sampling, periodic signals, reconstruction, recurrent nonuniform sampling, stability, uniform sampling.

\section{INTRODUCTION}

D IGITAL signal processing and image processing rely on sampling a continuous-time signal in order to obtain a discrete-time representation of the signal. Sampling theory has a long history and finds its roots in the work of Cauchy [1] and Gauss. Its name has almost become synonymous with that of $\mathrm{C}$. E. Shannon, who, amongst others, is credited with the statement of the uniform sampling theorem [2]. The Shannon-Whittaker theory states that from uniformly spaced samples, one may reconstruct a signal that contains no frequencies above half the sampling rate (a limit to which Nyquist's name has become attached). The importance and attraction of this theorem is that the samples of a bandlimited signal at an appropriate rate contain all information needed to reconstruct the signal.

Manuscript received October 25, 2006; revised December 5, 2007. This research was supported in part by the Israel Science Foundation under Grant 1081/07. The associate editor coordinating the review of this manuscript and approving it for publication was Dr. Hakan Johanson.

E. Margolis was with the Department of Electrical Engineering, Technion-Israel Institute of Technology, Haifa 32000, Israel. He is now with Freescale Semiconductor, Chandler, AZ 85224 USA (e-mail: evgeny.margolis@ gmail.com).

Y.C. Eldar is with the Department of Electrical Engineering, Technion-Israel Institute of Technology, Haifa 32000, Israel (e-mail: yonina@ee.technion.ac.il).

Color versions of one or more of the figures in this paper are available online at http://ieeexplore.ieee.org.

Digital Object Identifier 10.1109/TSP.2008.917416
Shannon's reconstruction theorem applies only to the case of uniform samples. There are a variety of applications in which the samples cannot be collected uniformly and the data are known only on a nonuniformly spaced sampling set. In communication theory, when data from a uniformly sampled signal are lost, the result is generally a sequence of nonuniform samples [3], [4]. The problem of signal reconstruction from its nonuniform frequency-domain samples arises in computerized tomography (CT) and magnetic resonance imaging (MRI) [5]. There are also applications where we can benefit from introducing irregular sampling (e.g., quantization methods [6]). In all of these examples, standard reconstruction techniques developed mainly for uniformly spaced samples [2] cannot be applied.

It is well established that a bandlimited signal is uniquely determined from its nonuniform samples, provided that the average sampling rate exceeds the Nyquist rate. The essential result is incorporated in the reconstruction theorem by Yao and Thomas [7]. Different extensions of the nonuniform sampling theorem are known [8]. Specifically, Yen considered the case where a finite number of uniform sampling points migrates in a uniform distribution to near distinct points [8]. He also considered the case of recurrent nonuniform sampling. A review of these reconstruction methods can be found in the tutorial article by Jerri [9]. Efficient filterbank reconstruction techniques from recurrent nonuniform samples were developed in [10].

Numerical implementation of all the reconstruction algorithms mentioned before is impossible on a digital computer due to practical limitations of the methods. Specifically, the reconstruction functions typically have infinite length. Thus, in practice, to overcome this problem, the infinite functions involved in the reconstruction must be truncated. In [11], finite-impulse response (FIR) filters were developed to reconstruct a nonperiodic-bandlimited signal from its recurrent nonuniform samples. Polynomial impulse-response FIR filters for reconstruction of two-periodic nonuniformly sampled signals were proposed in [12]. In both of these methods, perfect reconstruction is impossible; instead, the FIR filters are designed to optimize the least-squares and minimax reconstruction errors. Another issue is that in most practical applications, we only have a finite number of samples, while the reconstruction algorithms above require infinite data sets. Thus, the problem of reconstructing an infinite-length signal reduces to that of approximating the signal from a finite number of nonuniform samples.

In this paper, we consider the problem of reconstructing a periodic bandlimited signal from nonuniform samples. Given a finite number of samples, we can always extended them period- 
ically across the boundaries. Fortunately, in many practical situations, the functions under consideration are not arbitrary, but possess some smoothness properties, so that the signal can be regarded as approximately bandlimited. This approach is frequently encountered in image processing [13], where we are given a finite number of samples from a space-limited 2-D function. There are also applications, where the underlying signal is periodic. For example, a 2-D signal given in polar coordinates is periodic in its azimuthal coordinate [14].

Several iterative reconstruction methods for periodic bandlimited signals from nonuniform samples have been previously suggested [15], [16]. The method proposed by Marvasti in [15] is based on a sample and lowpass filter strategy in order to update the current solution. This strategy requires an accurate choice of the relaxation parameter and it is not always guaranteed to converge to the correct solution. An adaptive weights algorithm was presented in [16] as an alternative to Marvasti's approach to provide stable reconstruction. It turns out that this algorithm is sensitive to the sampling ratio of the reconstructed signal and may require a high number of iterations to converge. In [17], a noniterative block-based reconstruction approach was developed. This method requires the inversion of large complex-valued matrices and is designed to work only for special sets of nonuniform samples. In the case of an odd number of nonuniform samples, a closed-form (non iterative) reconstruction formula can be obtained using the Lagrange interpolation formula for trigonometric polynomials [18]. For an even number of samples, Lagrange interpolation for exponential polynomials results in a complex-valued interpolation function [19], [20]. What is lacking therefore is a closed-form method for reconstruction of a periodic bandlimited signal from an even number of nonuniform samples using real-valued functions. Furthermore, as we show in Section III, in the oversampled case, Lagrange interpolation provides reconstruction in a space which is larger than the space in which the original signal is contained. In noisy environments, this fact inserts perturbations to the reconstruction which are not in the space of the reconstructed signal (i.e., high-frequency components of the noise).

Our goal in this paper is to fill these gaps in the theory of nonuniform sampling of periodic bandlimited signals. Specifically, we derive closed-form algorithms involving real-valued functions for reconstructing a periodic bandlimited signal from an even and an odd number of nonuniform samples. We also develop reconstruction algorithms for oversampled periodic bandlimited signals, which provide reconstruction in the original signal space. In addition, we focus on some practical aspects of the reconstruction algorithms: stability and efficient implementation of the reconstruction. In [4], stability was discussed in the context of recovery of lost samples in nonperiodic bandlimited signals. In this paper, we provide a general framework for investigating the stability of noniterative methods for reconstruction of periodic bandlimited signals. The condition number is used to characterize the relation between the discrete-time noise of the samples and the resulting continuous-time error. We then apply our results to two specific sampling cases: uniform and recurrent nonuniform sampling, which are the most common in practical applications. We show that uniform sampling re- sults in the most stable and simple reconstruction algorithm. We note that similar results were derived in [21] for iterative reconstruction methods of periodic bandlimited signals. Recurrent nonuniform sampling is of interest in digital signal processing due to its ability to represent the sampling process as a bank of digital-to-analog (D-to-A) converters [22]. Here, we develop efficient filterbank structures for the reconstruction of periodic bandlimited signals from recurrent nonuniform samples.

This paper is organized as follows. In Section II, we start with a brief definition of frames and the space of periodic bandlimited signals. In Section III, we discuss the reconstruction of a periodic bandlimited signal from nonuniformly spaced samples. In Section IV, based on the theory of frames and the reconstruction method of Section III, we develop a new algorithm for the oversampled case. We then compare these two algorithms and discuss advantages, disadvantages, and properties of each method. Sections V and VI focus on the reconstruction of periodic bandlimited signals from uniform and recurrent nonuniform samples, respectively. In these sections, we derive simple reconstruction formulas and show that the stability analysis in these cases is simplified significantly. We then develop an efficient interpretation of the reconstruction processes from uniform and recurrent nonuniform samples using continuous-time linear time-invariant (LTI) filters. Experiments supporting our theoretical results and comparisons to standard methods found in the literature are provided in Section VII. A summary concluding the work is given in Section VIII. In the various sections, key results are stated and their detailed derivation is included in the appropriate Appendix.

\section{PRELIMINARY NOTIONS}

\section{A. Periodic Bandlimited Signals}

We consider the problem of reconstructing a periodic bandlimited signal from its nonuniform samples. A real periodic signal $x(t) \in L_{2}[0, T]$ with period $T$ that satisfies the Dirichlet conditions $^{1}$ has the following Fourier series representation:

$$
\begin{aligned}
x(t) & =\frac{a_{0}}{2}+\sum_{k=1}^{\infty}\left(a_{k} \cos \left(\frac{2 \pi k t}{T}\right)+b_{k} \sin \left(\frac{2 \pi k t}{T}\right)\right) \\
& =\sum_{k=-\infty}^{\infty} c_{k} \exp \left\{\frac{j 2 \pi k t}{T}\right\}
\end{aligned}
$$

where $L_{2}[0, T]$ is the space of square-integrable functions on the interval $[0, T]$ and $a_{k}, b_{k}, c_{k}$ are the Fourier coefficients of the trigonometric and exponential representations of $x(t)$, respectively, and are given by

$$
\begin{aligned}
a_{k} & =\frac{2}{T} \int_{0}^{T} x(t) \cos \left(\frac{2 \pi k t}{T}\right) \mathrm{dt} \\
b_{k} & =\frac{2}{T} \int_{0}^{T} x(t) \sin \left(\frac{2 \pi k t}{T}\right) \mathrm{dt} \\
c_{k} & =\frac{1}{T} \int_{0}^{T} x(t) \exp \left\{\frac{-j 2 \pi k t}{T}\right\} \mathrm{dt} .
\end{aligned}
$$

${ }^{1}$ The signal $x(t)$ is absolutely integrable over one period, has a finite number of maxima and minima in one period, and is continuous. 
The Fourier transform of $x(t)$ is defined as

$$
X(\omega)=\int_{-\infty}^{\infty} x(t) e^{-j \omega t} \mathrm{dt}=\sum_{k=-\infty}^{\infty} c_{k} \delta\left(\omega-\frac{2 \pi k}{T}\right)
$$

where $\delta(\omega)$ is the Dirac delta function.

A $T$-periodic signal $x(t)$ is said to be bandlimited to $2 \pi K / T$ if $c_{k}=0$ for $|k|>K$. Such signals are also known as trigonometric polynomials of degree $K$ [18]. We denote the space of $T$-periodic signals bandlimited to $2 \pi K / T$ by $V_{K}$ and refer to such signals as $K$-bandlimited. From (1), we conclude that $V_{K}$ is spanned by the $2 K+1$ orthonormal functions $\left\{e^{-j 2 \pi k t / T},-K \leqslant k \leqslant K\right\}$, namely

$$
V_{K}=\operatorname{span}\left\{e^{-j 2 \pi K t / T}, \ldots, 1, \ldots, e^{j 2 \pi K t / T}\right\} .
$$

The dimension of $V_{K}$ is $M=2 K+1$.

\section{B. Frames and Bases}

Our approach to reconstructing $x(t) \in V_{K}$ from its $N$ nonuniform samples $\left\{x\left(t_{i}\right)\right\}_{i=1}^{N}$ is to represent it as a linear combination of functions $\varphi_{i}(t) \in L_{2}[0, T]$, i.e.,

$$
x(t)=\sum_{i=1}^{N} x\left(t_{i}\right) \varphi_{i}(t)
$$

where the functions $\left\{\varphi_{i}(t)\right\}_{i=1}^{N}$ can be linearly independent, in which case they form a basis for the space $V_{K}$, or they can be linearly dependent, in which case they form a frame for the subspace $V_{K}$. Frames and bases provide a general framework for studying nonuniform sampling of periodic bandlimited signals [23]. The family of functions $\left\{\varphi_{i}(t)\right\}_{i=1}^{N} \in V(\varphi)$ is called a frame for $V(\varphi)$ if there exist constants $A>0$ and $B<\infty$ such that for all $x(t) \in V(\varphi)$ [24]

$$
A\|x(t)\|^{2} \leq \sum_{i=1}^{N}\left|\left\langle x(t), \varphi_{i}(t)\right\rangle\right|^{2} \leq B\|x(t)\|^{2} .
$$

Here, $\langle x(t), y(t)\rangle=(1 / T) \int_{0}^{T} x(t) y^{*}(t) d t$ is an inner product of two $T$-periodic functions $x(t)$ and $y(t)$ and $\|x(t)\|^{2}=\langle x(t), x(t)\rangle$ is the squared norm of $x(t)$. The constants $A$ and $B$ in (6) are called the frame bounds and $r=N / M$ is the redundancy, where $M$ is the dimension of $V(\varphi)$. If $N=M$ (i.e., $r=1$, then $\left\{\varphi_{i}(t)\right\}_{i=1}^{N}$ is a basis). If the two frame bounds are equal $A=B$, then the frame is called a tight frame. If, in addition, $N=M$, then $\left\{\varphi_{i}(t)\right\}_{i=1}^{N}$ is an orthonormal basis. The frame bounds can be determined as the highest and lowest eigenvalues of the frame correlation matrix $\mathbf{R}$, with the $i j$ th element

$$
\mathbf{R}_{i j}=\left\langle\varphi_{i}(t), \varphi_{j}(t)\right\rangle
$$

For a more comprehensive treatment of frames and bases, see, for example, [24].

\section{Stability Analysis}

One of the most important properties of a reconstruction algorithm is its stability, namely, the effect of a small perturbation of the samples on the reconstructed signal. If the samples
$\left\{x\left(t_{i}\right)\right\}_{i=1}^{N}$ of the signal $x(t)$ in (5) are perturbed by a sequence $\left\{w_{i}\right\}_{i=1}^{N}$, then, according to [25], the perturbation in the output $x_{w}(t)$ satisfies

$$
\sqrt{\frac{A}{B}} \frac{\|P w\|}{\left\|x\left(t_{p}\right)\right\|} \leq \frac{\left\|x_{w}(t)\right\|_{L_{2}[0, T]}}{\|x(t)\|_{L_{2}[0, T]}} \leq \sqrt{\frac{B}{A}} \frac{\|P w\|}{\left\|x\left(t_{p}\right)\right\|}
$$

where $P$ is an orthogonal projection onto the orthogonal complement of the null space of the frame operator, and $\|c\|^{2}=$ $1 / N \sum_{i=1}^{N} c_{i}^{2}$ denotes the squared norm of vector $c$ in an $N$-dimensional vector space. Based on the inequality (8), Unser and Zerubia [26] defined the condition number of the reconstruction algorithm as the ratio

$$
\kappa=\frac{B}{A}
$$

The quantity (9) provides an indicator of the stability and overall robustness of the reconstruction. The optimal situation is obviously $\kappa=1$, which holds in the case of an orthonormal basis or a tight frame. We observe from (7) that $\kappa$ depends on the distribution of the sampling points only and not on the sampled signal. This number may be very large in critical cases, where samples are very close to each other or large gaps exist between sampling points. As a result, the reconstruction algorithm becomes very unstable in these cases.

The calculation of $\kappa$ directly from the eigenvalues of the correlation matrix $\mathbf{R}$ can be computationally demanding for large values of $N$. In Sections V and VI, we develop methods for efficient evaluation of the resulting condition number for the case of uniform and recurrent nonuniform sampling.

\section{RECONSTRUCTION FROM NONUNIFORM SAMPLES}

The problem of reconstructing a $T$-periodic $K$-bandlimited signal $x(t)$ from its samples was first considered by Cauchy in 1841 [1] and was later investigated by several authors [19], [27]-[30].

A straightforward approach to reconstructing $x(t)$ of (1) from $N$ nonuniform samples $\left\{x\left(t_{p}\right)\right\}_{p=0}^{N-1}$ is to solve the set of $N$ linear equations with $2 K+1$ unknowns $\left\{c_{k}\right\}_{k=-K}^{K}$

$$
x\left(t_{p}\right)=\sum_{k=-K}^{K} c_{k} e^{j 2 \pi k t_{p} / T}, \quad p=0, \ldots, N-1 .
$$

Equation (10) can be expressed in matrix form as

$$
\mathbf{x}=\mathbf{A c}
$$

where the matrix $\mathbf{A}$ is given by

$$
\left(\begin{array}{ccccc}
e^{-j 2 \pi K t_{0} / T} & \cdots & 1 & \cdots & e^{j 2 \pi K t_{0} / T} \\
e^{-j 2 \pi K t_{1} / T} & \cdots & 1 & \cdots & e^{j 2 \pi K t_{1} / T} \\
\vdots & & \vdots & & \vdots \\
e^{-j 2 \pi K t_{N-1} / T} & \cdots & 1 & \cdots & e^{j 2 \pi K t_{N-1} / T}
\end{array}\right)
$$

and

$$
\begin{aligned}
\mathbf{x} & =\left(\begin{array}{lllll}
x\left(t_{0}\right) & x\left(t_{1}\right) & \cdots & x\left(t_{N-1}\right)
\end{array}\right)^{T} \\
\mathbf{c} & =\left(\begin{array}{lllll}
c_{-K} & \cdots & c_{0} & \cdots & c_{-K}
\end{array}\right)^{T}
\end{aligned}
$$


This method requires computing the inverse (if $N=2 K+1$ ) or pseudoinverse (if $N>2 K+1)$ of the $N \times(2 K+1)$ matrix $\mathbf{A}$ of (12), which is computationally demanding for large values of $N$. In addition to the computational complexity, this approach does not provide insight into the behavior of the reconstruction algorithm in noisy environments and we cannot use efficient signal-processing techniques, such as filtering and convolution in the reconstruction process.

Instead, we propose an alternative reconstruction strategy, where we directly represent the signal $x(t)$ as a linear combination of reconstruction functions $\left\{h_{p}(t)\right\}$, i.e.,

$$
x(t)=\sum_{p=0}^{N-1} x\left(t_{p}\right) h_{p}(t) .
$$

This method, together with the theory of frames, allows us to analyze the stability of the reconstruction algorithm and gain insight into the reconstruction process. It will also lead to efficient filter and filterbank structures for reconstruction from uniform and recurrent nonuniform samples, as we show in Sections V and VI.

The set of reconstruction functions $\left\{h_{p}(t)\right\}$ for $N=2 K+1$ uniform samples was first treated by Cauchy [1] and later by Stark [27], Brown [30], and Schanze [29] in different ways. Reconstruction from any even number $N$ of uniform samples was considered in [28] and [29]. As we show in Section V, these results are all special cases of Theorem 1 derived in this section.

Reconstructing a periodic bandlimited signal from nonuniform samples is considerably more complicated. When the number of sampling points $N$ is odd and it satisfies $N \geq 2 K+1$, $x(t)$ can be obtained using the Lagrange interpolation formula for trigonometric polynomials [18]. For any even $N$ greater than $2 K+1$, Lagrange interpolation generates a set of complex valued functions $\left\{h_{p}(t)\right\}$ [19], [20]. In Theorem 1, we will show that reconstruction can be obtained using real-valued functions that are simpler than those derived in [19].

Theorem 1: Let $x(t)$ be a $T$-periodic signal bandlimited to $2 \pi K / T$. Then, $x(t)$ can be perfectly reconstructed from its $N>$ $2 K+1$ even number of nonuniformly spaced samples $x\left(t_{p}\right)$ as

$$
x(t)=\sum_{p=0}^{N-1} x\left(t_{p}\right) h_{p}(t)
$$

where

$$
h_{p}(t)=\cos \left(\frac{\pi\left(t-t_{p}\right)}{T}\right) \prod_{\substack{q=0 \\ q \neq p}}^{N-1} \frac{\sin \left(\frac{\pi\left(t-t_{q}\right)}{T}\right)}{\sin \left(\frac{\pi\left(t_{p}-t_{q}\right)}{T}\right)} .
$$

For completeness, we recall the known result that reconstruction from $N \geq 2 K+1$ samples with $\mathrm{N}$ odd can also be obtained via (14) with [18]

$$
h_{p}(t)=\prod_{\substack{q=0 \\ q \neq p}}^{N-1} \frac{\sin \left(\frac{\pi\left(t-t_{q}\right)}{T}\right)}{\sin \left(\frac{\pi\left(t_{p}-t_{q}\right)}{T}\right)} .
$$

Proof: Let $\left\{t_{p}\right\}_{p=0}^{N-1}$ denote a set of nonuniformly distributed sampling points of a periodic bandlimited signal $x(t)$ such that $0 \leq t_{0}<\cdots<t_{N-1}<T$, where $T$ is the period of $x(t)$. Using the periodic property of the signal (i.e., $x\left(t_{p}\right)=x\left(t_{p}+n T\right)$ ), we see that arbitrary sampling of a periodic signal corresponds to a recurrent nonuniform sampling scheme of nonperiodic signals according to which a group of $N$ samples repeats itself along the nonperiodic signal with period $T$. A formula for reconstructing a bandlimited signal from recurrent nonuniform samples was derived by Yen in [8] and is given by

$x(t)=\sum_{n=-\infty}^{\infty} \sum_{p=0}^{N-1} x\left(t_{p}+n T\right) \frac{a_{p}(-1)^{n N} \prod_{q=0}^{N-1} \sin \left(\frac{\pi\left(t-t_{q}\right)}{T}\right)}{\frac{\pi\left(t-n T-t_{p}\right)}{T}}$

where

$$
a_{p}=\prod_{\substack{q=0 \\ q \neq p}}^{N-1} \frac{1}{\sin \left(\frac{\pi\left(t_{p}-t_{q}\right)}{T}\right)} .
$$

Here, the average sampling rate should be greater or equal than the Nyquist rate (i.e., $N \geq 2 K+1$ ). Substituting the nonuniform samples $x\left(t_{p}\right)=x\left(t_{p}+n T\right)$ into (16), we have

$$
x(t)=\sum_{p=0}^{N-1} x\left(t_{p}\right) \sum_{n=-\infty}^{\infty} \frac{a_{p}(-1)^{n N} \prod_{q=0}^{N-1} \sin \left(\frac{\pi\left(t-t_{q}\right)}{T}\right)}{\frac{\pi\left(t-n T-t_{p}\right)}{T}} .
$$

We now define the function $h_{p}(t)$ as the function which multiplies the $p$ th sampled value in (18). Using the relation $\sin (t-$ $n \pi)=(-1)^{n} \sin (t)$, we can express $h_{p}(t)$ as

$$
\begin{aligned}
h_{p}(t) & =\sum_{n=-\infty}^{\infty} \frac{a_{p}(-1)^{n N} \prod_{q=0}^{N-1} \sin \left(\frac{\pi\left(t-t_{q}\right)}{T}\right)}{\frac{\pi\left(t-n T-t_{p}\right)}{T}} \\
& =\sum_{n=-\infty}^{\infty} a_{p} \frac{\prod_{q=0}^{N-1} \sin \left(\frac{\pi\left(t-n T-t_{q}\right)}{T}\right)}{\frac{\pi\left(t-n T-t_{p}\right)}{T}} .
\end{aligned}
$$

It is easily seen that $h_{p}(t)$ is periodic in $T$ and satisfies the Dirichlet conditions and, therefore, has the following Fourier series representation:

$$
h_{p}(t)=\sum_{k=-\infty}^{\infty} c_{k} e^{j 2 \pi k t / T}
$$

where

$$
c_{k}=\frac{1}{T} \int_{0}^{T} h_{p}(t) e^{-j 2 \pi k t / T} \mathrm{dt} .
$$

Substituting $h_{p}(t)$ into (21), we have

$$
\begin{aligned}
c_{k}=\frac{a_{p}}{T} \int_{0}^{T} \sum_{n=-\infty}^{\infty} \frac{\sin \left(\frac{\pi\left(t-n T-t_{p}\right)}{T}\right)}{\frac{\pi\left(t-n T-t_{p}\right)}{T}} \\
\quad \times \prod_{\substack{q=0 \\
q \neq p}}^{N-1} \sin \left(\frac{\pi\left(t-n T-t_{q}\right)}{T}\right) e^{-j 2 \pi k t / T} \mathrm{dt} .
\end{aligned}
$$


To simplify (22), we replace $t$ by a new variable $u=t-n T-t_{p}$. The integration limits are changed accordingly to $I_{n}=[-n T-$ $\left.t_{p}, T-n T-t_{p}\right]$. Using the fact that $e^{-j 2 \pi k n}=1$ for any integers $n$ and $k,(22)$ reduces to

$$
\begin{aligned}
c_{k}=\frac{a_{p}}{T} e^{-j 2 \pi k t_{p} / T} \sum_{n=-\infty}^{\infty} \int_{I_{n}} \frac{\sin \left(\frac{\pi u}{T}\right)}{\frac{\pi u}{T}} \\
\times \prod_{\substack{q=0 \\
q \neq p}}^{N-1} \sin \left(\frac{\pi\left(u+t_{p}-t_{q}\right)}{T}\right) e^{-j 2 \pi k u / T} \mathrm{du} .
\end{aligned}
$$

In Appendix A, we explicitly calculate the Fourier coefficients $c_{k}$ of (23) and show that for $N$ even

$$
c_{k}= \begin{cases}a_{p} \frac{b_{2 k-1}+b_{2 k+1}}{2} e^{-j 2 \pi k t_{p} / T}, & |k| \leq \frac{N}{2} \\ 0, & \text { otherwise }\end{cases}
$$

where the complex coefficients $b_{l}$ are the result of expanding the product of sines in (78). As we will see, the exact values of $b_{l}$ do not matter, as they will cancel out in the final expression.

Substituting (24) into (20)

$$
\begin{aligned}
h_{p}(t)=\frac{a_{p}}{2} & \sum_{k=-N / 2}^{N / 2} b_{2 k-1} e^{j 2 \pi k\left(t-t_{p}\right) / T} \\
& +\frac{a_{p}}{2} \sum_{k=-N / 2}^{N / 2} b_{2 k+1} e^{j 2 \pi k\left(t-t_{p}\right) / T} .
\end{aligned}
$$

Denoting $l_{1}=2 k-1$ and $l_{2}=2 k+1$ in the first and the second terms of (25), respectively, and using the property (80) of the coefficient set $b_{l}$ with the fact that $b_{N+1}=b_{-N-1}=0$, we have

$$
\begin{aligned}
h_{p}(t)= & \frac{a_{p}}{2} e^{j \pi\left(t-t_{p}\right) / T} \sum_{l_{1}=-N+1}^{N-1} b_{l_{1}} e^{j \pi l_{1}\left(t-t_{p}\right) / T} \\
& +\frac{a_{p}}{2} e^{-j \pi\left(t-t_{p}\right) / T} \sum_{\substack{l_{2}=-N+1 \\
N-1}}^{N l_{2} e^{j \pi l_{2}\left(t-t_{p}\right) / T}} \\
= & \frac{a_{p}}{2} e^{j \pi\left(t-t_{p}\right) / T} \prod_{\substack{q=0 \\
q \neq p}}^{N-1} \sin \left(\frac{\pi\left(t-t_{q}\right)}{T}\right) \\
& +\frac{a_{p}}{2} e^{-j \pi\left(t-t_{p}\right) / T} \prod_{\substack{q=0 \\
q \neq p}}^{N-1} \sin \left(\frac{\pi\left(t-t_{q}\right)}{T}\right) \\
= & \cos \left(\frac{\pi\left(t-t_{p}\right)}{T}\right) \prod_{\substack{q=0 \\
q \neq p}}^{N-1} \frac{\sin \left(\frac{\pi\left(t-t_{q}\right)}{T}\right)}{\sin \left(\frac{\pi\left(t_{p}-t_{q}\right)}{T}\right)}
\end{aligned}
$$

completing the proof of the theorem.

Note that the Lagrange interpolation formula of (15b) can be obtained by applying mathematical derivations, similar to (25) and (26), for the case of $N$ odd.

In the next subsection, we discuss properties of the reconstruction algorithm proposed by Theorem 1 .

\section{A. Properties of the Reconstruction Functions}

Combining (20) and (24), the reconstruction functions $\left\{h_{p}(t)\right\}$ of (15) can be expanded into finite sums of complex exponential functions

$$
h_{p}(t)=\sum_{l=-\lfloor N / 2\rfloor}^{\lfloor N / 2\rfloor} c_{p l} e^{j 2 \pi l t / T}
$$

where $\lfloor\cdot\rfloor$ denotes the floor operator, which rounds down to the nearest integer. This representation will be used in Section IV to develop an alternative reconstruction algorithm for nonuniformly oversampled periodic bandlimited signals. From (27), we conclude that the functions $h_{p}(t)$ are periodic in $T$ and have a limited number of harmonics (i.e., they are bandlimited). Theorem 2 will establish that they are also linearly independent and define the space of periodic bandlimited signals for which the set of functions $\left\{h_{p}(t)\right\}_{p=0}^{N-1}$ constitutes a basis.

Theorem 2: The set of reconstruction functions $\left\{h_{p}(t)\right\}_{p=0}^{N-1}$ defined by (15a) for $N$ even constitutes a basis for the space $V$, where

$$
V=V_{(N-2) / 2} \bigcup \sin \left(\frac{\pi\left(N t-\sigma_{t}\right)}{T}\right) .
$$

Here, $\sigma_{t}=\sum_{p=0}^{N-1} t_{p}$, and $V_{K}$ is the space of $T$-periodic $K$-bandlimited functions.

Proof: To prove Theorem 2, we have to show that the set of $N$ functions $\left\{h_{p}(t)\right\}_{p=0}^{N-1}$ of (15a) spans the $N$-dimensional space $V$ of (28). It follows directly from Theorem 1 that $V_{(N-2) / 2}$ is in the span of $\left\{h_{p}(t)\right\}_{p=0}^{N-1}$. We also observe that the function $x_{s}(t)=\sin \left(\pi\left(N t-\sigma_{t}\right) / T\right)$ is orthogonal to any function in $V_{(N-2) / 2}$. Therefore, the dimension of the space $V$ itself is $N$ and we need to show that the function $x_{s}(t)$ is also in the span of $\left\{h_{p}(t)\right\}_{p=0}^{N-1}$. In terms of Theorem 1, we have to prove that

$$
x_{s}(t)=\sum_{p=0}^{N-1} x_{s}\left(t_{p}\right) h_{p}(t) .
$$

Substituting $x_{s}(t)$ and the exponential expansions of the functions $\left\{h_{p}(t)\right\}_{p=0}^{N-1}$, which are given in (27) into (29), we have

$$
x_{s}(t)=\sum_{p=0}^{N-1} x_{s}\left(t_{p}\right) \sum_{l=-N / 2}^{N / 2} c_{p l} e^{j 2 \pi l t / T} .
$$

Since $x_{s}(t)$ is a sine function with frequency $\pi N / T$, only the terms with $|l|=N / 2$ in (30) contribute to the reconstruction of the signal $x_{s}(t)$. Using this fact, (30) can be rewritten as

$$
\sum_{p=0}^{N-1} x_{s}\left(t_{p}\right)\left(c_{p(-N / 2)} e^{-j \pi N t / T}+c_{p(N / 2)} e^{j \pi N t / T}\right) .
$$

It can be shown that the complex coefficients $c_{p(-N / 2)}$ and $c_{p(N / 2)}$ are given by [31, App. C]

$$
c_{p(N / 2)}=c_{p(-N / 2)}^{*}=\frac{a_{p}(-1)^{(N / 2-1)}}{j 2^{N}} e^{-j \pi \sigma_{t} / T}
$$


and the constant $a_{p}$ is defined in (17). Substituting (32) into (31), results in

$$
\frac{(-1)^{(N / 2-1)}}{2^{N-1}} \sum_{p=0}^{N-1} x_{s}\left(t_{p}\right) \frac{a_{p}}{j 2}\left(e^{j \pi\left(N t-\sigma_{t}\right) / T}-e^{-j \pi\left(N t-\sigma_{t}\right) / T}\right) .
$$

The last expression can be simplified to

$$
x_{s}(t)=\sin \left(\frac{\pi\left(N t-\sigma_{t}\right)}{T}\right) \frac{(-1)^{(N / 2-1)}}{2^{N-1}} \sum_{p=0}^{N-1} a_{p} x_{s}\left(t_{p}\right) .
$$

Theretofore, our problem reduces to the proof of the following trigonometric identity:

$$
\sum_{p=0}^{N-1} a_{p} \sin \left(\frac{\pi\left(N t_{p}-\sigma_{t}\right)}{T}\right)=(-1)^{(N / 2-1)} 2^{N-1} .
$$

Substituting constants $a_{p}$ and $\sigma_{t}$ into (35), we have

$$
\sum_{p=0}^{N-1} \frac{\sin \left(\sum_{q=0, q \neq p}^{N-1}\left(\frac{\pi\left(t_{p}-t_{q}\right)}{T}\right)\right)}{\prod_{q=0, q \neq p}^{N-1} \sin \left(\frac{\pi\left(t_{p}-t_{q}\right)}{T}\right)}=(-1)^{(N / 2-1)} 2^{N-1} .
$$

The proof of (36) is given in Appendix B.

From Theorem 2, we observe that the functions $\left\{h_{p}(t)\right\}_{p=0}^{N-1}$ do not span a complete space of periodic bandlimited signals. To complete this space to $V_{N / 2}$, we need to add any function of the form $\sin (\pi(N t-\theta) / T)$, where $\theta \neq \sigma_{t}+T n$ and $n$ is an integer. Note, that in the case of $N$ odd, the reconstruction functions of (15b) span a complete space $V_{(N-1) / 2}$.

An important observation from Theorem 2 is that in the oversampled case (i.e., $N>2 K+1$ ), the functions $\left\{h_{p}(t)\right\}$ span a space which is larger than the space $V_{K}$ containing the signal $x(t)$ that is sampled. This fact is the basis for the development of an alternative reconstruction method in Section IV.

We can immediately verify that the reconstruction functions $\left\{h_{p}(t)\right\}_{p=0}^{N-1}$ of Theorem 1 have the interpolation property, namely

$$
h_{p}\left(t_{k}\right)=\left\{\begin{array}{ll}
1, & k=p, \\
0, & k \neq p,
\end{array} \quad k, p=0,1, \ldots, N-1 .\right.
$$

If $x(t)$ is not bandlimited, then the reconstruction $\widehat{x}(t)$ given by Theorem 1 is not equal to $x(t)$. Nonetheless, the interpolation property (37) guaranties consistent reconstruction of the signal $x(t)$ (i.e., $\widehat{x}\left(t_{p}\right)=x\left(t_{p}\right)$ ). Consistency is an important property for many signal/image-processing applications [25]. One such application is the recovery of missing samples from the set of remaining samples [3], [4]. In this application, consistency guarantees the desirable property that the set of the remaining samples stays unchanged after reconstruction.

In the next section, we develop an alternative reconstruction algorithm for oversampled periodic bandlimited signals, where the set of reconstruction functions constitutes a frame.

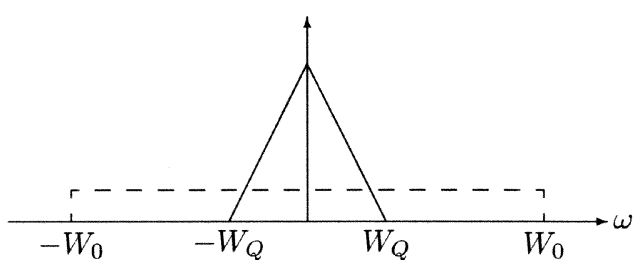

Fig. 1. Power spectrum of a signal (solid line) and white noise (dashed line).

\section{FRAME-BASED RECONSTRUCTION}

\section{A. Philosophy of the Approach}

Theorem 1 in Section III provides perfect reconstruction of periodic bandlimited signals from nonuniform redundant $(N>$ $2 K+1)$ and nonredundant $(N=2 K+1)$ samples. From Theorem 2, it follows that in the case of redundant sampling, the set of reconstruction functions proposed by Theorem 1 spans a space which is larger than the space of the signal $V_{K}$. Thus, the fact that the signal is oversampled is not taken into account in the reconstruction process (i.e., the narrow band signal bandlimited to $2 \pi K / T$ is reconstructed with functions of wider support).

One of the main reasons for oversampling is to reduce the average power of additive noise or quantization error, in the samples $\left\{x\left(t_{p}\right)\right\}$, [32], [33]. To clarify how the redundancy may contribute to the reconstruction process, we consider uniform sampling of a nonperiodic signal $f(t)$ bandlimited to $W_{Q}$. The Shannon-Wittaker theory guarantees perfect reconstruction of such signals from uniformly spaced samples taken at the Nyquist interval $T_{Q}=\pi / W_{Q}$, where $\operatorname{sinc}\left(t / T_{Q}\right)$ is chosen as the reconstruction function [2]. Reconstruction with $\operatorname{sinc}\left(t / T_{Q}\right)$ is equivalent to ideal low-pass filtering in the frequency domain, where the support of the filter is $\left[-W_{Q}, W_{Q}\right]$. In the oversampled case in which the sampling period is $T_{0}<T_{Q}$, according to the Shannon-Wittaker theory, perfect reconstruction is obtained with an ideal low-pass filter of support $[-W, W]$, where any $W_{Q} \leq W \leq W_{0}$ with $W_{0}=\pi / T_{0}$ will lead to perfect reconstruction. When the samples $f_{n}=f\left(n T_{0}\right)$ of the signal are corrupted by white noise (e.g., as a result of quantization [33]), we obtain only an approximated version of the signal $f(t)$. Fig. 1 shows the power spectral density of the signal bandlimited to $W_{Q}$ and white noise. From Fig. 1, we can immediately conclude that the optimal choice is to use a low-pass filter with support $\left[-W_{Q}, W_{Q}\right]$. This was mathematically proved in [32, p. 137], where it was also shown that the set of shifted versions of the function $\operatorname{sinc}\left(t / T_{Q}\right)$ constitutes a tight frame for the space of signals bandlimited to $W_{Q}$. This approach is advantageous in analog-to-digital conversion techniques, where it is practically easier to increase the sampling rate than to reduce the quantization error (i.e., quantization step size [34]).

This oversampling example is easily analyzed and understood without frame formalism. However, in more complicated representations, the frame approach is needed. For example, framebased algorithms for redundant sampling and reconstruction in arbitrary spaces were proposed in [35]. 


\section{B. Reconstruction From Nonuniform Samples Using Frames}

Consider now the reconstruction of a periodic bandlimited signal. In the oversampled case, if the samples are corrupted by noise, then applying a low-pass filter of support $[-2 \pi K / T, 2 \pi K / T]$ to the reconstructed signal can reduce the average power of the noise. We denote this low-pass filtering as an operator $P_{K}$, which zeros all harmonics higher than $K$ of the periodic signal $x(t)$. Formally

$$
P_{K} x(t)=\sum_{k=-K}^{K} c_{k} e^{j 2 \pi k t / T}
$$

where $x(t)$ is a $T$-periodic signal that is not necessarily bandlimited. We can immediately show that $P_{K}$, defined in (38), is an orthogonal projection of a $T$-periodic signal $x(t)$ onto the subspace of $T$-periodic $K$-bandlimited signals $V_{K}$. Applying $P_{K}$ to the reconstructed signal of (14) results in

$$
x(t)=P_{K} \sum_{p=0}^{N-1} x\left(t_{p}\right) h_{p}(t)=\sum_{p=0}^{N-1} x\left(t_{p}\right) P_{K} h_{p}(t)
$$

which is equivalent to a reconstruction with the set of functions $\left\{P_{K} h_{p}(t)\right\}$. Computing these functions explicitly leads to the following reconstruction theorem. Since the proof is tedious, we do not provide it here, but rather refer the reader to [31, Theor. 4.1].

Theorem 3: The problem of reconstructing a $T$-periodic $K$-bandlimited signal from arbitrary spaced samples, considered in Theorem 1, can be solved by (14), with

$h_{p}(t)=\frac{\alpha_{p 0}}{2}+\sum_{k=1}^{K}\left(\alpha_{p k} \cos \left(\frac{2 \pi k t}{T}\right)-\beta_{p k} \sin \left(\frac{2 \pi k t}{T}\right)\right)$.

For $N$ odd

$$
\begin{aligned}
& \alpha_{p k}=\frac{a_{p}(-1)^{k}}{2^{N-2}} \sum_{\varphi \in G_{p k}} \cos \left(\frac{\pi \varphi}{T}\right) \\
& \beta_{p k}=\frac{a_{p}(-1)^{k}}{2^{N-2}} \sum_{\varphi \in G_{p k}} \sin \left(\frac{\pi \varphi}{T}\right)
\end{aligned}
$$

where $G_{p k}$ is the set of all possible sums of values $\left\{t_{q}\right\}_{q=0, q \neq p}^{N-1}$, when $(N-1) / 2+k$ of them are chosen with a negative sign and $(N-1) / 2-k$ are positive.

For $N$ even

$$
\begin{gathered}
\alpha_{p k}=\frac{a_{p}(-1)^{k}}{2^{N-1}}\left(\sum_{\varphi \in G_{p k}^{+}} \sin \left(\frac{\pi \varphi}{T}\right)-\sum_{\varphi \in G_{p k}^{-}} \sin \left(\frac{\pi \varphi}{T}\right)\right) \\
\beta_{p k}=\frac{a_{p}(-1)^{k}}{2^{N-1}}\left(\sum_{\varphi \in G_{p k}^{-}} \cos \left(\frac{\pi \varphi}{T}\right)-\sum_{\varphi \in G_{p k}^{+}} \cos \left(\frac{\pi \varphi}{T}\right)\right)
\end{gathered}
$$

where the set $\left\{G_{p k}^{+} \oplus G_{p k}^{-}\right\}$consists of all possible sums of values $\left\{t_{q}\right\}_{q=0}^{N-1}$, when $N / 2+k$ of them are chosen with the
TABLE I

SUMMARY AND COMPARISON OF TwO RECONSTRUCTION METHODS

\begin{tabular}{l|c|c}
\hline \hline & Theorem 1 & Theorem 3 \\
\hline \hline Perfect reconstruction in $V_{K}$ & Yes & Yes \\
Interpolation property & Yes & No \\
Set of reconstruction functions forms & Basis & Frame \\
Stability of reconstruction & Less stable & More stable \\
\hline \hline
\end{tabular}

negative sign and $N / 2-k$ are positive. The value of $t_{p}$ appears with positive and negative signs in $G_{p k}^{+}$and $G_{p k}^{-}$, respectively.

The explicit formulas for calculating the coefficients $\alpha_{p k}$ and $\beta_{p k}$ of (41) and (42) are obtained by applying the orthogonal projection $P_{K}$ of (38) onto (27). To determine the properties and relation of these reconstruction methods, we rely on the following proposition.

1) Proposition 1: ([24, p. 95]): Let $\left\{\varphi_{i}(t)\right\}_{i=1}^{N}$ be a Riesz basis for the space $W$ with frame bounds $A_{W}$ and $B_{W}$, and let $P$ denote the orthogonal projection of $W$ onto a closed subspace $V$. Then, $\left\{P \varphi_{i}(t)\right\}_{i=1}^{N}$ is a frame for $V$ with frame bounds $A_{V}$ and $B_{V}$, such that $A_{V} \geq A_{W}$ and $B_{V} \leq B_{W}$.

Proposition 1 leads directly to the conclusion that the set of functions $\left\{P_{K} h_{p}(t)\right\}$ of (40) constitutes a frame for the space $V_{K}$ with redundancy ratio $r=N /(2 K+1)$. From Proposition 1, we may also conclude that the frame bounds of the set $\left\{P_{K} h_{p}(t)\right\}$ are tighter (no looser) than the bounds of the set $\left\{h_{p}(t)\right\}$ of (15). As a result, the condition number $\kappa=A / B$ of the frame $\left\{P_{K} h_{p}(t)\right\}$ is no larger than $\kappa$ of $\left\{h_{p}(t)\right\}$.

In the ideal case in which $x(t)$ is $K$-bandlimited and its samples are not corrupted by noise, the reconstruction functions (15) and (40) both lead to perfect reconstruction of $x(t)$. However, when $x(t)$ is not truly bandlimited or its samples $\left\{x\left(t_{p}\right)\right\}$ are corrupted by noise, these two methods lead to different reconstructions. Note that the interpolation property (37) no longer holds when using (40) (i.e., in the nonideal case, the reconstructed signal $\widehat{x}\left(t_{p}\right)$ does not necessarily satisfy $\left.\widehat{x}\left(t_{p}\right)=x\left(t_{p}\right)\right)$.

To conclude this section, we summarize the basic properties of the reconstruction functions $\left\{h_{p}(t)\right\}$ developed in Theorem 3 and compare them to the properties of the reconstruction functions of Theorem 1 in Table I.

In the following sections, we consider two special cases of sampling: Uniform and recurrent nonuniform sampling.

\section{RECONSTRUCTION FROM UNIFORM SAMPLES}

The most popular form of sampling used in the context of digital signal processing (DSP) is uniform sampling in which $t_{p}=p T / N$, due to its simplicity and stability of reconstruction. The formulas for reconstructing a periodic bandlimited signal from uniform odd, even, and redundant samples were already developed in [1], [27], [29], and [30]. In this section, we show that these results are all special cases of Theorems 1 and 3. We then analyze the stability of the reconstruction algorithms.

\section{A. Reconstruction Formulas}

To simplify the expression for $h_{p}(t)$ of Theorem 1 for this case, we first show that product of sines in (15) has an equiva- 
lent representation, which holds only for the case of uniformly spaced data

$$
\prod_{\substack{q=0 \\ q \neq p}}^{N-1} \frac{\sin \left(\frac{\pi\left(t-t_{q}\right)}{T}\right)}{\sin \left(\frac{\pi\left(t_{p}-t_{q}\right)}{T}\right)}=\frac{\sin \left(\frac{N \pi\left(t-t_{p}\right)}{T}\right)}{N \sin \left(\frac{\pi\left(t-t_{p}\right)}{T}\right)} .
$$

The proof of (43) is given in Appendix C. The last expression is a scaled Dirichlet kernel $D_{N}(t)$ [18], which plays an important role in the theory of DSP. Substituting (43) into (15), we have

$$
h_{p}(t)= \begin{cases}\frac{\sin \left(\frac{N \pi\left(t-t_{p}\right)}{T}\right)}{N \sin \left(\frac{\pi\left(t-t_{p}\right)}{T}\right)}, & N \text { odd } \\ \cos \left(\frac{\pi\left(t-t_{p}\right)}{T}\right) \frac{\sin \left(\frac{N \pi\left(t-t_{p}\right)}{T}\right)}{N \sin \left(\frac{\pi\left(t-t_{p}\right)}{T}\right)}, & N \text { even }\end{cases}
$$

which is equal to the reconstruction function derived in [1], [27], [30], and [29].

In Appendix D, we show that for the uniformly oversampled periodic bandlimited signal, the frame-based reconstruction function is given by

$$
h_{p}(t)=\frac{\sin \left(\frac{\pi(2 K+1)\left(t-t_{p}\right)}{T}\right)}{N \sin \left(\frac{\pi\left(t-t_{p}\right)}{T}\right)}
$$

which is equal to the reconstruction function derived in [28] in a different way.

\section{B. Reconstruction With an LTI Filter}

For uniform samples, the reconstruction (14) can be expressed as $x(t)=s(t) * h(t)$, where $s(t)$ is an impulse train of samples

$$
s(t)=\sum_{p=0}^{N-1} x\left(t_{p}\right) \delta\left(t-t_{p}\right)
$$

and

$$
h(t)= \begin{cases}\frac{\sin \left(\frac{N \pi t}{T}\right)}{N \sin \left(\frac{\pi t}{T}\right)}, & N \text { odd } \\ \cos \left(\frac{\pi t}{T}\right) \frac{\sin \left(\frac{N \pi t}{T}\right)}{N \sin \left(\frac{\pi t}{T}\right)}, & N \text { even. }\end{cases}
$$

From (46), it follows that $x(t)$ is obtained by filtering $s(t)$ with an LTI filter with an impulse response $h(t)$ given by (47). This filtering operation is schematically shown in Fig. 2(a).

The frequency response $H(\omega)$ of the continuous-time filter $h(t)$ of (47) is given by (48), shown at the bottom of the page, which was obtained by calculating the Fourier transform of (47).

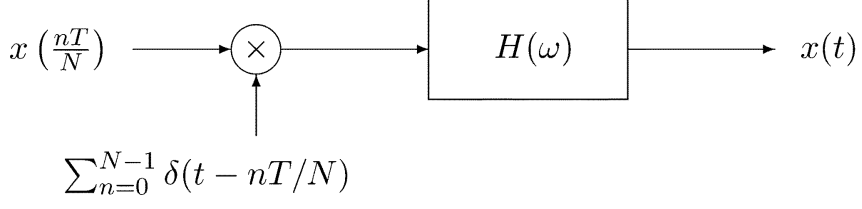

(a)

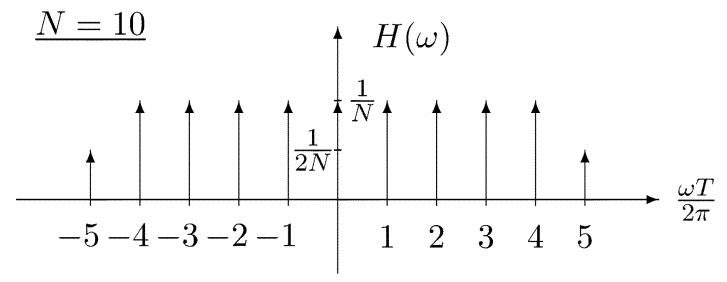

(b)

Fig. 2. Reconstruction from uniform samples using a continuous-time filter (a) Filtering scheme. (b) Frequency response of the filter for $N=10$.

The frequency response $H(\omega)$ of (48) for the case $N=10$ (even) is shown in Fig. 2(b).

Evidently, in both cases of odd and even number $N$, the filters are bandlimited. For $N$ odd, $H(\omega)$ is a low-pass filter (LPF) for periodic uniformly sampled signals with a cutoff frequency of $\pi(N-1) / T$. In the case of $N$ even, $H(\omega)$ is wider than the LPF with cutoff frequency $\pi(N-2) / T$ but is not an LPF with cutoff $\pi N / T$. For the frame-based reconstruction of (45), $H(\omega)$ is an LPF with cutoff frequency $2 \pi K / T$.

\section{Stability of Reconstruction With Bases}

We now analyze the stability of the reconstruction functions (44), based on the calculation of the correlation matrix $\mathbf{R}$, defined in (7). Due to the special structure of $\mathbf{R}$ for uniformly spaced samples, the calculation of its eigenvalues and the condition number $\kappa$ are immediate.

In the case of $N$ odd, the entry $(i, k)$ of the correlation matrix $\mathbf{R}$ (i.e., the inner product of two periodic functions $h_{i}(t)$ and $\left.h_{k}(t)\right)$ is given by $\left\langle h_{i}(t), h_{k}(t)\right\rangle=(1 / N) \delta(i-k)$, or equivalently, $\mathbf{R}=(1 / N) I_{N \times N}$, where $I_{N \times N}$ is the $N \times N$ identity matrix. Therefore, for $N$ odd, the set of reconstruction functions of (44) constitutes an orthonormal basis for the space $V_{(N-1) / 2}$, and its condition number is $\kappa=1$, which is the lowest possible value for $\kappa$. Due to the low condition number, the set $\left\{h_{p}(t)\right\}$ of (44) provides stable reconstruction in the presence of noise. Results for the case of $N$ even, are summarized in the following theorem.

$$
H(\omega)= \begin{cases}\frac{1}{N} \sum_{n=-(N-1) / 2}^{(N-1) / 2} \delta\left(\omega-\frac{2 \pi n}{T}\right), & N \text { odd } \\ \frac{1}{2 N} \delta\left(\omega-\frac{N \pi}{T}\right)+\frac{1}{2 N} \delta\left(\omega+\frac{N \pi}{T}\right)+\frac{1}{N} \sum_{n=-(N-2) / 2}^{(N-2) / 2} \delta\left(\omega-\frac{2 \pi n}{T}\right), & N \text { even }\end{cases}
$$


Theorem 4: The set of reconstruction functions of (44) for $N$ even constitutes a basis for the space $V$, where $V=V_{(N-2) / 2} \bigcup \cos (\pi N t / T)$ and its condition number is $\kappa=2$.

Proof: The first statement is obtained from substituting the set of uniform samples into (28) and using the fact that $\sin (\theta-$ $\pi / 2)=\cos (\theta)$.

To prove that $\kappa=2$, calculating the inner products of the functions $h_{i}(t)$ and $h_{k}(t)$, we have

$$
\mathbf{R}_{i k}=\left\{\begin{array}{cl}
\frac{2 N-1}{2 N_{1}^{2}}, & i=k \\
\frac{1}{2 N^{2}}, & (i-k) \text { odd } \\
-\frac{1}{2 N^{2}}, & (i-k) \text { even and } i \neq k
\end{array}\right.
$$

From (49), $\mathbf{R}$ is a symmetric circulant matrix of the form

$$
\mathbf{R}=\left(\begin{array}{ccccc}
a & b & -b & \cdots & b \\
b & a & b & \cdots & -b \\
-b & b & a & & b \\
\vdots & \vdots & & \ddots & \vdots \\
b & -b & b & \cdots & a
\end{array}\right)
$$

where $b=1 /\left(2 N^{2}\right)$ and $a=1 / N-b$. Denoting the $N$ values of the first row of the matrix $\mathbf{R}$ by $r_{n}, n=0, \ldots, N-1$, we have $\mathbf{R}_{i k}=r_{(k-i) \bmod N}$. Since $\mathbf{R}$ is circulant, its eigenvalues $\left\{\lambda_{m}\right\}_{m=0}^{N-1}$ can be easily calculated as the discrete Fourier transform of $r_{n}$ [36]

$$
\lambda_{m}=\sum_{n=0}^{N-1} r_{n} e^{-j 2 \pi n m / N} .
$$

Substituting the values of the first row of $\mathbf{R}$ into (51)

$$
\begin{aligned}
\lambda_{m} & =a+b-b \sum_{n=0}^{N-1} e^{j \pi n} e^{-j 2 \pi n m / N} \\
& = \begin{cases}a-(N-1) b, & m=\frac{N}{2} \\
a+b, & m \neq \frac{N}{2}\end{cases}
\end{aligned}
$$

The condition number $\kappa$ is then

$$
\kappa=\frac{a+b}{a-(N-1) b}=2 .
$$

From Theorem 4, we conclude that the set of functions $\left\{h_{p}(t)\right\}$ is not an orthonormal basis, for which $\kappa=1$. We also observe that $\kappa$ does not depend on $N$ and $T$; thus, the set never converges to an orthonormal basis. As a result, reconstruction from an even number of uniform samples, where $\kappa=2$, may be less stable compared to $N$ odd, where $\kappa=1$ and the set $\left\{h_{p}(t)\right\}$ is orthonormal.

\section{Stability of Reconstruction With Frames}

Our main result concerning the reconstruction with frames is stated in the following theorem.

Theorem 5: The set of reconstruction functions of (45) constitutes a tight frame for the space $V_{K}$.

Proof: See [31, p. 58].

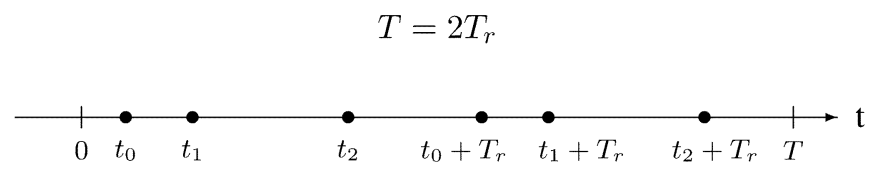

Fig. 3. Recurrent nonuniform sampling distribution for $N_{r}=3$ and $M_{r}=2$.

From Theorem 5, we conclude that frame-based reconstruction with functions (45) leads to the lowest possible condition number $\kappa=1$. Since the number of reconstruction functions is $N$ and the dimension of the space $V_{K}$ is $2 K+1$, the redundancy ratio $r$ of this tight frame is $N /(2 K+1)$.

Reconstruction with frames is also popular due to its ability to remove the high-frequency components of the noise. It was shown in [32] that for the case of reconstruction of nonperiodic bandlimited signals from uniform samples that the power of white additive noise is reduced by a factor of $r$. This result holds also for uniform sampling of periodic bandlimited signals. The theoretical derivations of this section will be supported by simulation results in Section VII.

\section{RECURRENT NONUNIFORM SAMPLING}

We now consider the case of recurrent nonuniform sampling. In this form of sampling, the sampling points are divided into groups of $N_{r}$ nonuniformly spaced points. The groups have a recurrent period, which is denoted by $T_{r}$. One group of nonuniform samples repeats itself $M_{r}$ times along the $T$-periodic signal $x(t)$, where $M_{r} T_{r}=T$. Denoting the points in the first recurrent group by $t_{r}, r=0,1, \ldots N_{r}-1$, the complete set $\left\{t_{p}\right\}_{p=0}^{N-1}$ of sampling points in one period $T$ is

$$
t_{p} \in\left\{t_{r}+n T_{r}, r=0, \ldots N_{r}-1, n=0, \ldots M_{r}-1\right\}
$$

or equivalently

$$
t_{p}=t_{p \bmod N_{r}}+\left\lfloor\frac{p}{N_{r}}\right\rfloor T_{r}, \quad p=0,1, \ldots N-1 .
$$

An example of a sampling distribution for the case $N_{r}=3$ and $M_{r}=2$ is depicted in Fig. 3 .

This sampling scheme can be regarded as a combination of $N_{r}$ sequences of uniform samples with $M_{r}$ points each, taken with interval $T_{r}$. This interpretation of the sampling method is schematically shown in Fig. 4. Recurrent nonuniform sampling arises in a variety of applications, among them conversion of continuous-time signals to discrete time using a set of $N_{r}$ A-to-D converters, each operating at the rate $1 / T_{r}$. In the general case, these $N_{r}$ converters are not necessarily synchronized, leading to a recurrent nonuniform distribution of the samples. From a practical point of view, it is much easier to design a set of $N_{r}$ converters each with rate $1 / T_{r}$ than one converter working at the higher rate of $N_{r} / T_{r}$.

We first develop a formula for reconstructing a periodic bandlimited signal from recurrent nonuniform samples. We then develop efficient implementations of this reconstruction formula using continuous-time and discrete-time LTI filters and analyze the stability of the reconstruction method. 


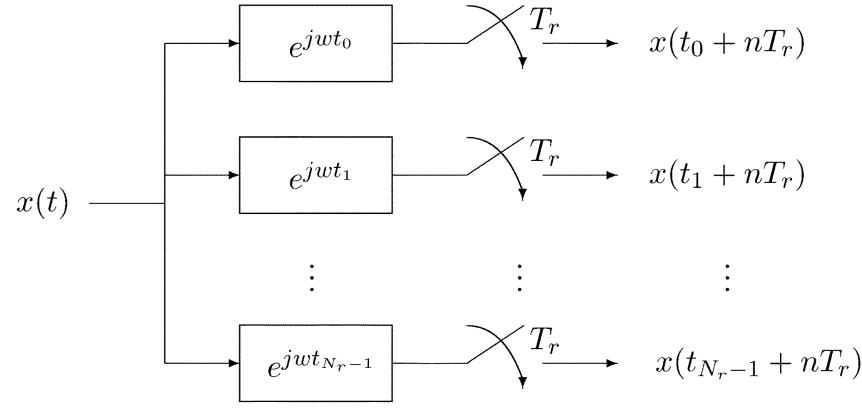

Fig. 4. Recurrent nonuniform sampling model.

\section{A. Derivation of the Reconstruction Functions}

Substituting the recurrent nonuniform sampling set of (54) into (15b) for an odd number of samples (i.e., $N=M_{r} N_{r}$ is odd), and using the identity (43) which is satisfied for equally spaced samples and the fact that $\sin (\pi(t-\theta) / T)=-\sin (\pi(t-$ $\theta-T) / T)$, we have

$$
\begin{aligned}
h_{p}(t)= & \prod_{m=1}^{M_{r}-1} \frac{\sin \left(\frac{\pi\left(t-t_{p}-m T_{r}\right)}{T}\right)}{\sin \left(\frac{\pi\left(t_{p}-t_{p}-m T_{r}\right)}{T}\right)} \\
& \times \prod_{q=0}^{N_{r}-1} \prod_{m=0}^{M_{r}-1} \frac{\sin \left(\frac{\pi\left(t-t_{q}-m T_{r}\right)}{T}\right)}{\sin \left(\frac{\pi\left(t_{p}-t_{q}-m T_{r}\right)}{T}\right)} \\
= & \frac{\sin \left(\frac{M_{r} \pi\left(t-t_{p}\right)}{T}\right)}{M_{r} \sin \left(\frac{\pi\left(t-t_{p}\right)}{T}\right)} \prod_{q=0}^{N_{r}-1} \frac{\sin \left(\frac{M_{r} \pi\left(t-t_{q}\right)}{T}\right)}{\sin \left(\frac{M_{r} \pi\left(t_{p}-t_{q}\right)}{T}\right)} \\
= & b_{p} \frac{\prod_{q=0}^{N_{r}-1} \sin \left(\frac{M_{r} \pi\left(t-t_{q}\right)}{T}\right)}{\sin \left(\frac{\pi\left(t-t_{p}\right)}{T}\right)}
\end{aligned}
$$

where

$$
b_{p}=\frac{1}{M_{r} \prod_{q=0, q \neq p}^{N_{r}-1} \sin \left(\frac{M_{r} \pi\left(t_{p}-t_{q}\right)}{T}\right)} .
$$

Similarly, we can derive the reconstruction function for an even number of samples, which results in

$$
h_{p}(t)=b_{p} \cos \left(\frac{\pi\left(t-t_{p}\right)}{T}\right) \frac{\prod_{q=0}^{N_{r}-1} \sin \left(\frac{M_{r} \pi\left(t-t_{q}\right)}{T}\right)}{\sin \left(\frac{\pi\left(t-t_{p}\right)}{T}\right)}
$$

where $b_{p}$ is defined by (57).

From the definition of the reconstruction functions of (56) and (58), we can immediately verify that if two functions $h_{i}(t)$ and $h_{j}(t)$ belong to one of the $N_{r}$ uniform sets (see Fig. 4) (i.e., $i-j=m N_{r}$ where $m$ is an integer), then one function is a shifted version of the other, namely

$$
h_{p}(t)=h_{\left(p \bmod N_{r}\right)}\left(t-\left\lfloor\frac{p}{N_{r}}\right\rfloor T_{r}\right) .
$$

The frame-based reconstruction from recurrent nonuniform oversampling of periodic bandlimited signals is obtained by orthogonal projection of the set $\left\{h_{p}(t)\right\}$ of (56) or (58) onto the space $V_{K}$. We do not give an explicit formula for this set of functions due to the complexity of representation, but we discuss its properties and provide efficient implementations of the reconstruction in the following subsections.

\section{B. Reconstruction Using a Continuous-Time Filterbank}

We now show that the reconstruction formulas (56) and (58) from recurrent nonuniform samples can be implemented using a continuous time filterbank. The ideas of this section are similar to those developed in [10] for recurrent nonuniform sampling of nonperiodic signals.

First, using the periodicity of the recurrent nonuniform sampling and the property (59), which states that all of the functions in the set (56) or (58) are shifted versions of $N_{r}$ functions, (14) can be rewritten as

$$
x(t)=\sum_{n=0}^{M_{r}-1} \sum_{p=0}^{N_{r}-1} x\left(t_{p+n N_{r}}\right) h_{p}\left(t+n T_{r}\right) .
$$

The last expression (60) can be equivalently represented as a sum of $N_{r}$ convolutions

$$
x(t)=\sum_{p=0}^{N_{r}-1} s_{p}(t) * h_{p}(t)
$$

where $s_{p}(t)$ is an impulse train of samples

$$
s_{p}(t)=\sum_{n=0}^{M_{r}-1} x\left(n T_{r}+t_{p}\right) \delta\left(x-n T_{r}-t_{p}\right) .
$$

For $N$ odd

$$
h_{p}(t)=b_{p} \frac{\prod_{q=0}^{N_{r}-1} \sin \left(\frac{M_{r} \pi\left(t+t_{p}-t_{q}\right)}{T}\right)}{\sin \left(\frac{\pi t}{T}\right)}
$$

and for $N$ even

$$
h_{p}(t)=b_{p} \cos \left(\frac{\pi t}{T}\right) \frac{\prod_{q \neq p}^{N_{r}-1} \sin \left(\frac{M_{r} \pi\left(t+t_{p}-t_{q}\right)}{T}\right)}{\sin \left(\frac{\pi t}{T}\right)}
$$

where $b_{p}$ is defined in (57). Equation (61) can be interpreted as a continuous-time filterbank as depicted in Fig. 5. Each of the $N_{r}$ uniform sequences of samples $s_{p}(t)$ formed according to (62) is filtered by a continuous-time filter $H_{p}(\omega)$ with an impulse response given by (63). Summing the outputs of the $N_{r}$ filters results in the reconstructed signal $x(t)$.

Note that each subsequence corresponds to uniform samples at one- $N_{r}$ th of the average sampling rate. Therefore, the output of each branch of the filter bank is an aliased and filtered version of $x(t)$. The filters, as specified by (63), have the inherent property that the aliasing components of the filter outputs cancel in forming the summed output $x(t)$. 


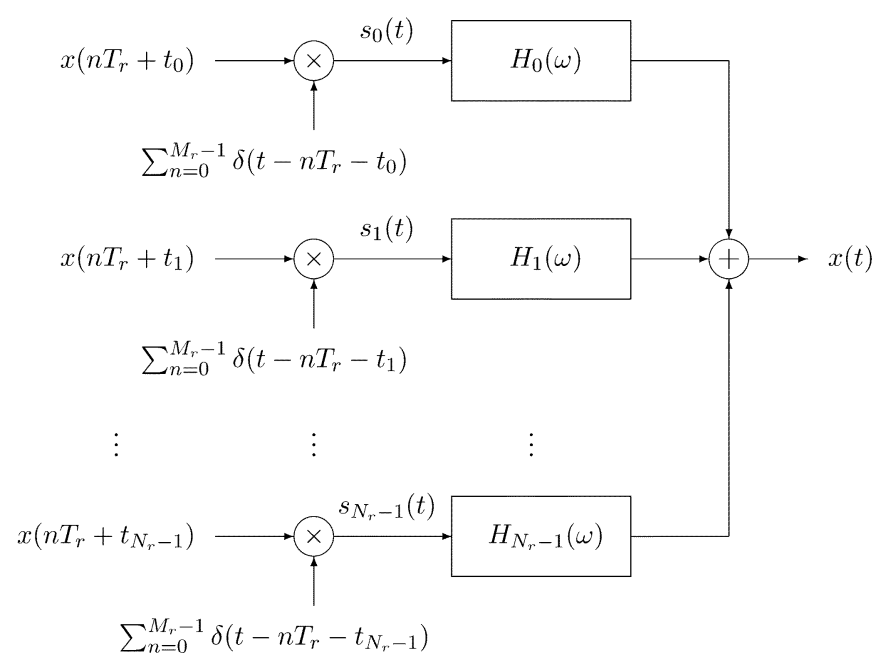

Fig. 5. Reconstruction from recurrent nonuniform samples using a continuoustime filterbank.

To determine the frequency response $H_{p}(\omega)$ of the filter $h_{p}(t)$ of (63), we note that $h_{p}(t)$ can be expressed as

$$
h_{p}(t)=b_{p} \frac{\sin \left(\frac{M_{r} \pi t}{T}\right)}{\sin \left(\frac{\pi t}{T}\right)} \sum_{k=-N_{r}+1}^{N_{r}-1} c_{p k} e^{j k M_{r} \pi t / T}
$$

and for $N$ even

$$
h_{p}(t)=b_{p} \cos \left(\frac{\pi t}{T}\right) \frac{\sin \left(\frac{M_{r} \pi t}{T}\right)}{\sin \left(\frac{\pi t}{T}\right)} \sum_{k=-N_{r}+1}^{N_{r}-1} c_{p k} e^{j k M_{r} \pi t / T}
$$

where the complex coefficients $c_{p k}$ are the result of expanding the product of sines in (63) into complex exponentials.

The first terms in (64) correspond to LPFs with cutoff frequency $\pi M_{r} / T$, which we denote as $H_{\mathrm{LPF}}\left(\omega ; \pi M_{r} / T\right)$. The effect of the summation and multiplexing by the exponent is to create $N_{r}$ shifted and scaled versions of this bandlimited filter, i.e.,

$$
H_{p}(\omega)=b_{p} \sum_{k=-N_{r}+1}^{N_{r}-1} c_{p k} H_{\mathrm{LPF}}\left(\omega-\frac{k \pi M_{r}}{T} ; \frac{\pi M_{r}}{T}\right) .
$$

Hence, we conclude that the filters $H_{p}(\omega)$ in Fig. 5 are bandlimited to $\pi M_{r} N_{r} / T$, and each filter $H_{p}(\Omega)$ is piecewise constant over frequency intervals of length $2 \pi M_{r} / T$. This fact allows for further efficiency in the implementation.

Note that the filter frequency responses $H_{p}(\omega)$ for periodic signals are the sampled versions of the filter frequency responses for the nonperiodic case treated in [10]. This fact is illustrated in Fig. 6 of the following example.

1) Example 1: We consider the problem of reconstructing a periodic ten-bandlimited signal $x(t)$ from recurrent nonuniform samples with $T=2 \pi$. The set of nonuniform samples in the first group is given by $t_{0}=0, t_{1}=0.087$, and $t_{2}=0.227$, repeated with period $T_{r}=\pi / 6$ so that $N_{r}=3$ and $M_{r}=12$.

The reconstruction in this case is obtained using a bank of three continuous time filters. In Fig. 6(a), we depict the third filter $H_{2}(\omega)$ of the filterbank. As we expect, the filter is bandlimited to $\pi M_{r} N_{r} / T=12 \cdot 3 / 2=18$, since $H_{2}(\omega)$ is created

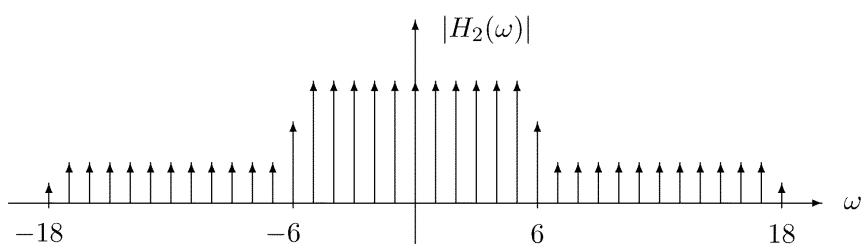

(a)

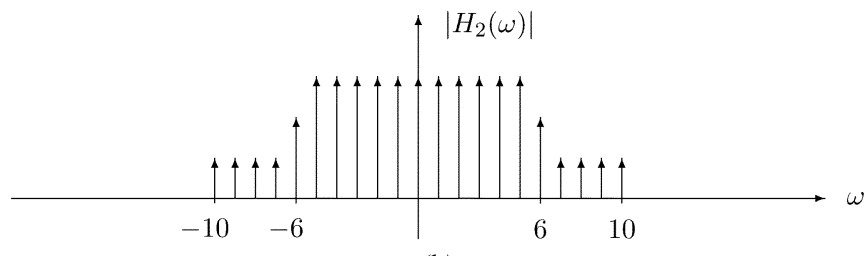

(b)

Fig. 6. Frequency response of $H_{2}(\omega)$ for the reconstruction methods of Theorem 1 (a) and Theorem 3 (b).

by three $\left(N_{r}\right)$ shifted and scaled versions of a filter bandlimited to $M_{r} / 2=6$.

We note that using the frame-based reconstruction also leads to a filterbank reconstruction, similar to that described in this section. In Fig. 6(b), we depict the third filter $H_{2}(\omega)$ of the filterbank of the reconstruction method of Theorem 3. As expected, this filter is an orthogonally projected version of the filter depicted in Fig. 6(a) onto the space of the reconstructed signal.

\section{Interpolation and Reconstruction Using a Discrete-Time Filterbank}

Most of today's signal-processing operations are performed on digital computers. Therefore, efficient techniques for digital processing of the signal are required. Following an analogous procedure in [10], the continuous-time filterbank of Fig. 5 can be converted to a discrete-time filterbank followed by a continuoustime LPF.

The interpolation identity, which was developed in [10], states that each branch of the continuous-time filterbank of Fig. 5 can be replaced by an equivalent block. This block consists of expanding a sequence of samples by a factor of $N_{r}$ and then filtering by a discrete-time filter

$$
\widetilde{H}_{p}(\omega)=\frac{N}{T} H_{p}\left(\frac{N \omega}{T}\right) e^{-j t_{p} N \omega / T}, \quad|\omega| \leq \pi .
$$

The filter output is then followed by impulse modulation and lowpass filtering.

Applying this interpolation identity to each branch in Fig. 5 and moving the impulse modulation and LPF in each branch outside the summer, we obtain the equivalent implementation in Fig. 7.

The discrete-time filterbank of Fig. 7 can be used to interpolate the uniform samples and to reconstruct the continuous-time signal from its recurrent nonuniform samples very efficiently, exploiting the many known results regarding the implementation of filterbank structures. We also note that due to the nature of periodic signals, each filter in Fig. 7 is of finite length. Therefore, these filters can be exactly calculated by combining (64) and (66) and there is no need to approximate these filters as in the case of infinite length filterbank structures. As with the 


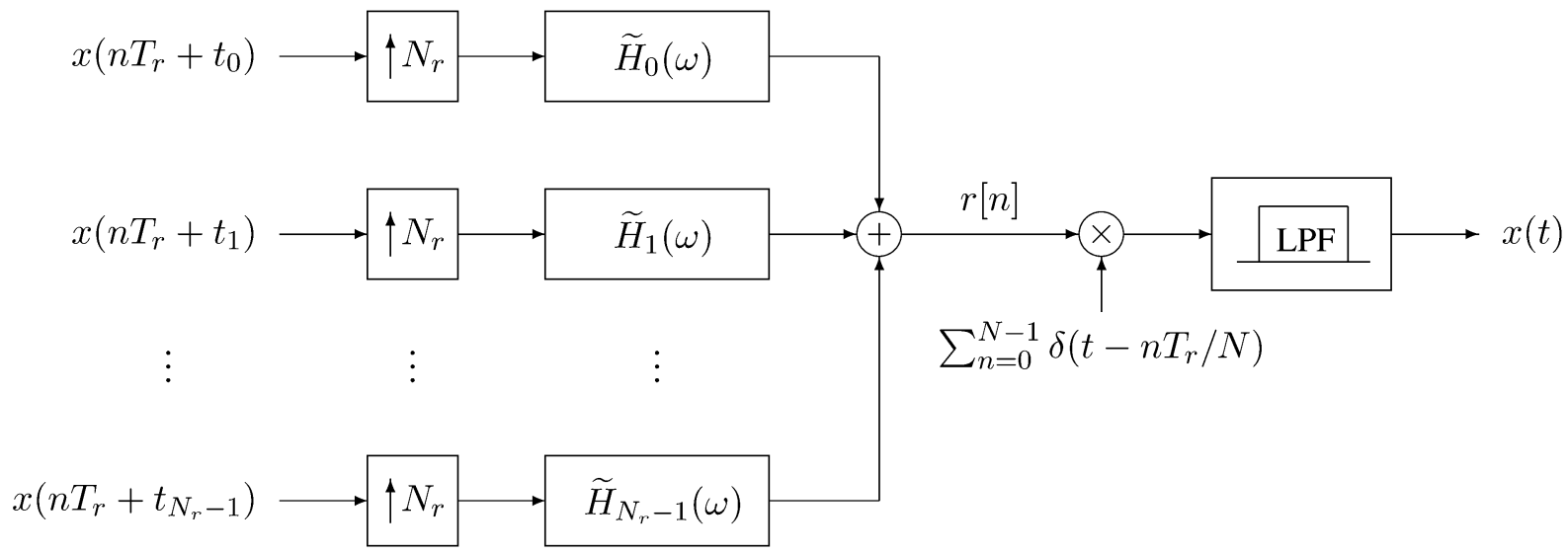

Fig. 7. Reconstruction from recurrent nonuniform samples by using a discrete-time filterbank.

continuous-time filterbank, the magnitude response of the discrete-time filters is piecewise constant, which allows for further efficiency in the implementation.

\section{Stability of Reconstruction}

We now provide an analysis of the stability of the reconstruction, using the set of reconstruction functions $\left\{h_{p}(t)\right\}$ of (58). Similar to the case of uniform sampling, we calculate the correlation matrix $\mathbf{R}$ of the set $\left\{h_{p}(t)\right\}$ and provide an efficient technique for determining its eigenvalues. We also present an example of recurrent nonuniform samples and investigate the behavior of the condition number as a function of the sampling distribution. As we will see, the optimal distribution of the samples, which results in the lowest value of $\kappa$ in the case of bandlimited signals, is uniform.

Using (59) and the fact that the inner product of two periodic functions $x(t)$ and $y(t)$ is shift invariant, namely $\langle x(t-\tau), y(t-$ $\tau)\rangle=\langle x(t), y(t)\rangle$, we can show that

$$
\mathbf{R}(i, j)=\mathbf{R}\left(\left(i+n N_{r}\right) \bmod N,\left(j+n N_{r}\right) \bmod N\right) .
$$

From (67), we conclude that the matrix $\mathbf{R}$ has the following form:

$$
\mathbf{R}=\left(\begin{array}{ccccc}
\mathbf{A}_{0} & \mathbf{A}_{1} & \mathbf{A}_{2} & \cdots & \mathbf{A}_{M_{r}-1} \\
\mathbf{A}_{M_{r}-1} & \mathbf{A}_{0} & \mathbf{A}_{1} & \cdots & \mathbf{A}_{M_{r}-2} \\
\vdots & \vdots & & \ddots & \vdots \\
\mathbf{A}_{1} & \mathbf{A}_{2} & & \cdots & \mathbf{A}_{0}
\end{array}\right)
$$

where the submatrices $\mathbf{A}_{r}$ and $r=0, \ldots, M_{r}-1$ are $N_{r} \times N_{r}$ matrices with entries

$$
\mathbf{A}_{r}(i, j)=\left\langle h_{i}(t), h_{j+r N_{r}}(t)\right\rangle
$$

Evidently, $\mathbf{R}$ is a block circulant matrix, where each row of submatrices is a cyclic shift of the row above it [37]. To compute the eigenvalues of $\mathbf{R}$, we define the discrete Fourier components of $\mathbf{R}$ as

$$
\widehat{\mathbf{A}}_{k}=\sum_{r=0}^{M_{r}-1} \mathbf{W}^{k r} \mathbf{A}_{r}, \quad k=0, \ldots, M_{r}-1
$$

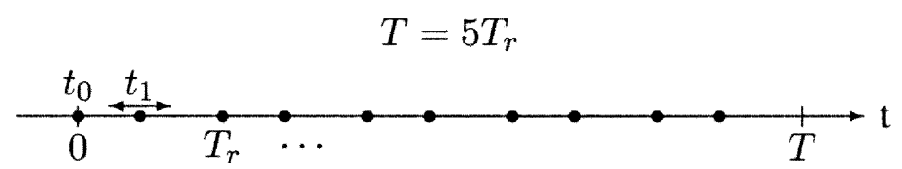

Fig. 8. Sampling distribution for $N_{r}=2$ and $M_{r}=5$.

where $\mathbf{W}=e^{-j\left(2 \pi / M_{r}\right)}$. Let $\left\{\lambda_{k, i}\right\}_{i=0}^{N_{r}-1}$ be the $N_{r}$ eigenvalues of $\widehat{\mathbf{A}}_{k}$ for every $k=0, \ldots, M_{r}-1$. It was shown in [37] that the eigenvalues of an Hermitian block circulant matrix are the eigenvalues of the discrete Fourier components. Therefore, the eigenvalues of the matrix $\mathbf{R}$ of (68) are given by

$$
\lambda(\mathbf{R})=\lambda_{k, i}, \quad i=0, \ldots N_{r}-1, k=0, \ldots M_{r}-1 .
$$

The last result (71), significantly simplifies the calculation of the condition number $\kappa$ of the reconstruction algorithm in the case of recurrent nonuniform samples. Instead of computing $N$ eigenvalues of an $N \times N$ matrix $\mathbf{R}$, which may be very large, we compute $N_{r}$ eigenvalues of the $M_{r}$ matrices $\widehat{\mathbf{A}}_{k}$ defined by (70), where typically $N_{r} \ll N$. We note that for the framebased reconstruction, the computation of the condition number is exactly the same.

The following example emphasizes the simplicity of computing $\kappa$ in the case of recurrent nonuniform samples. In this example, we compare the condition number $\kappa$, when a basis and frame are used for reconstruction. We also show that the optimal distribution of the samples, which results in the lowest possible value of the condition number, is uniform.

1) Example 2: We consider a recurrent nonuniform set of samples, where $N_{r}=2, M_{r}=5$, and the period of the reconstructed signal is $T=10$. The remaining parameters can be calculated as $T_{r}=T / M_{r}=2$ and $N=N_{r} M_{r}=10$ (even). Without loss of generality, we assume throughout this example that $t_{0}=0$. Thus, the sample $t_{1}$, which dictates the position of the second uniform sequence of samples, may take on values in the range $0<t_{1}<T_{r}$. We define the space of the $T$-periodic signal $x(t)$ involved in reconstruction to be $V_{2}$ (i.e., the redundancy of the frame is $r=10 / 5=2$ ). An example of this set of samples is presented in Fig. 8, where the vector illustrates the mobility of the sample $t_{1}$. 


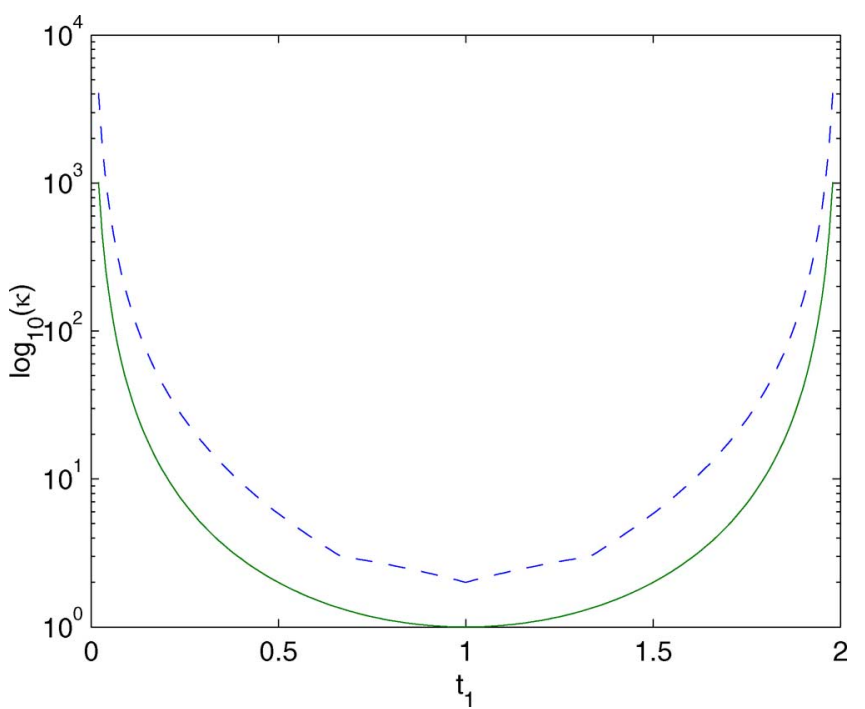

Fig. 9. Comparison between the condition number $\kappa$ of the reconstruction method of Theorem 1 (dashed line) and of the frame method of Theorem 3 (solid line), as a function of $t_{1}$. The set of recurrent nonuniform samples is defined in Example 2 and the space of the sampled signal is $V_{2}(r=2)$.

The behavior of the condition number as a function of the location of $t_{1}$ is shown in Fig. 9. The smallest and largest eigenvalues are obtained from (71) by computing the eigenvalues of $52 \times 2$ matrices, which is much easier than direct calculation of the eigenvalues of the $10 \times 10$ matrix $\mathbf{R}$.

As predicted by Proposition 1 , the condition number of the frame method is significantly lower than $\kappa$ of the set of reconstruction functions of (58). We observe, that in the neighborhood of $t_{1}=1$, the condition number of the frame based method is very low and it achieves the minimal value for $t_{1}=1$, where the recurrent nonuniform sampling set becomes uniform and the set of reconstruction functions constitutes a tight frame. We also note that in the reconstruction method of (58), the lowest achievable value of $\kappa$ for this sampling set is 2 , as predicted by (53) for $N$ even.

From this example, we conclude that uniform sampling results in the most stable behavior of the reconstruction algorithms of Theorems 1 and 3. Analogous results were presented by Ferreira in [21] for the case of reconstructing a periodic bandlimited signal from nonuniform samples using iterative algorithms. Specifically, it was shown that the best convergence rates of the iterative approaches result when the distances between the samples are equal.

Fig. 9 provides a good tool for the practical design of sampling and reconstruction systems. By determining the maximal value for $\kappa$, which we are able to tolerate in our reconstruction system, we can find the minimal-allowed shifts between the sets of uniform samples in the recurrent nonuniform sampling scheme. Given this information, we can prevent critical (nonstable) distributions of samples.

\section{Simulations}

In this section, we complete the theoretical discussions of Sections III - VI by simulating the proposed reconstruction algorithms. Specifically, we present reconstruction examples for the methods of Theorems 1 and 3 in a noisy environment. We
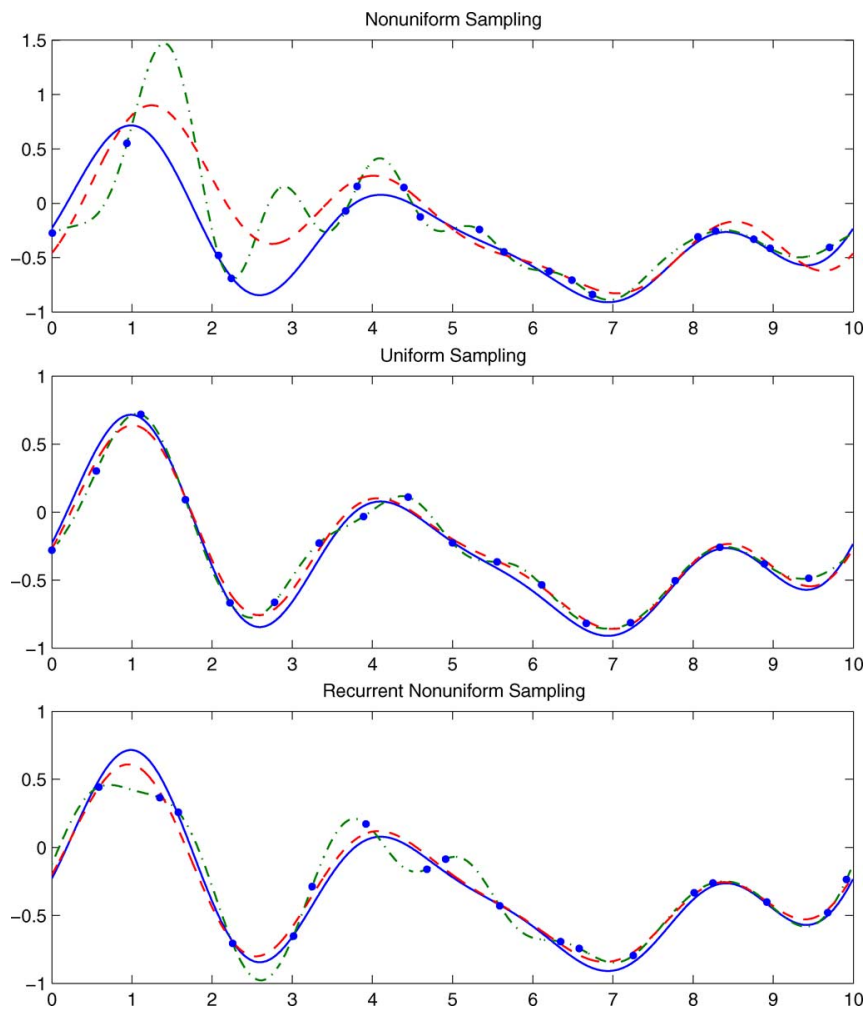

Fig. 10. Reconstruction of a ten-periodic four-bandlimited signal (solid line) from 18 nonuniform (top), uniform (middle), and recurrent nonuniform (bottom) noisy samples (marked by dots) using the reconstruction methods of Theorem 1 (dashed-dotted line) and Theorem 3 (dashed line).

then compare their performance to other algorithms for both cases, with and without noise. Finally, we demonstrate the performance on reconstruction of nonperiodic bandlimited signals.

\section{A. Reconstruction Example}

Our first example illustrates the reconstruction of a periodic bandlimited signal from nonuniform, uniform, and recurrent nonuniform noisy samples using the two reconstruction algorithms of Theorems 1 and 3.

We first created in Matlab a ten-periodic four-bandlimited signal $x(t)$, which belongs to the 9-D space $V_{4}$. Then, three sets of 18 sampling points $\left\{t_{p}\right\}$ were considered: 1$)$ nonuniform; 2) uniform; and 3) recurrent nonuniform with $N_{r}=3$, where the nonuniform points were randomly chosen. The redundancy of these sampling sets is $r=2$. Each sample $x\left(t_{p}\right)$ was perturbed by zero-mean white Gaussian noise $w_{p}$ with variance 0.01 . We define the indicator of the reconstruction quality as a relative mean square error (MSE), given by

$$
\mathrm{MSE}=\frac{\|x(t)-\widehat{x}(t)\|_{L_{2}[0, T]}}{\|x(t)\|_{L_{2}[0, T]}}
$$

where $\widehat{x}(t)$ is the reconstructed signal.

The reconstruction results of $x(t)$ from nonuniform, uniform, and recurrent nonuniform noisy samples are shown in Fig. 10 in the top, middle, and bottom plots, respectively. The MSE values are summarized in Table II.

We can clearly see from Fig. 10 and Table II that the reconstructed signal $\widehat{x}(t)$ is closer to the original signal $x(t)$ when the 
TABLE II

Relative Mean Square ERror of Two Reconstruction Methods for DifFerent SETS OF SAMPLING POINTS

\begin{tabular}{c|ccc}
\hline \hline Reconstruction & \multicolumn{3}{|c}{ MSE [dB] } \\
\cline { 2 - 4 } Method & Nonuniform & Uniform & Recurrent Nonuniform \\
\hline \hline Theorem 1 & -3.62 & -16.84 & -13.08 \\
Theorem 3 & -6.35 & -19.87 & -19.82 \\
\hline \hline
\end{tabular}

frame method of Theorem 3 is used. An important observation is that the dashed-doted curve passes through the noisy samples. This result was expected, since the set of reconstruction functions of Theorem 1 satisfies the interpolation property (37).

In Table II, we see that the frame method results in the same reconstruction quality for uniform and recurrent nonuniform sampling sets. Obviously, we expect less stable behavior in the case of recurrent nonuniform samples. In this example, a large portion of the noise energy was removed by orthogonal projection (8).

\section{B. Comparison of the Reconstruction Methods With Noise}

We now compare our approach to standard methods found in the literature. Specifically, we consider three algorithms: The first is an iterative method proposed by Marvasti in [15], based on the following iteration:

$$
x_{k}(t)=x_{k-1}(t)+\lambda S\left(x(t)-x_{k-1}(t)\right)
$$

where $\lambda, x(t)$, and $x_{k}(t)$ are a relaxation parameter, the original signal, and the result of the $k$ th iteration with $x_{0}(t)=0$, respectively. The low-pass filter $S$ is used to update the current iteration. In our simulations, we chose $\lambda=0.1$ and the reconstruction is obtained after 20 iterations.

The second approach is an iterative adaptive weights method that was developed in [16]. The adaptive weight value $\omega_{p}$ of the $p$ th sample is calculated as an average distance to the closest sample from each side. The idea is to solve the equation $\mathbf{T}_{w} \mathbf{a}_{w}=\mathbf{y}_{w}$ by utilizing the method of conjugate gradients for the solution of the Toeplitz-type structure of the system matrix, where

$$
\mathbf{T}_{w}(k, l)=\frac{1}{T} \sum_{p=0}^{N-1} \omega_{p} e^{-j 2 \pi(k-l) t_{p} / T}, \quad \text { for }|l|,|k| \leq K
$$

and the vector $y_{w}$ is given by

$$
\mathbf{y}_{w}(k)=\frac{1}{\sqrt{T}} \sum_{p=0}^{N-1} x\left(t_{p}\right) \omega_{p} e^{-j 2 \pi k t_{p} / T}, \quad \text { for }|k| \leq 2 K+1 .
$$

The solution $\mathbf{a}_{w}$ is taken after 20 iterations of the algorithm. The reconstruction is then given by $x(t)=$ $1 / \sqrt{T} \sum_{n=-K}^{K} \mathbf{a}_{w}(n) e^{j 2 \pi n t_{p} / T}$.

The last method we consider is a block-based approach proposed by Tuncer in [17]. This algorithm can only be used when the sampling points have the form

$$
t_{p}=\left(p+\frac{\tau_{p}}{L}\right) \frac{T}{N}, \quad p=0,1, \ldots N-1
$$

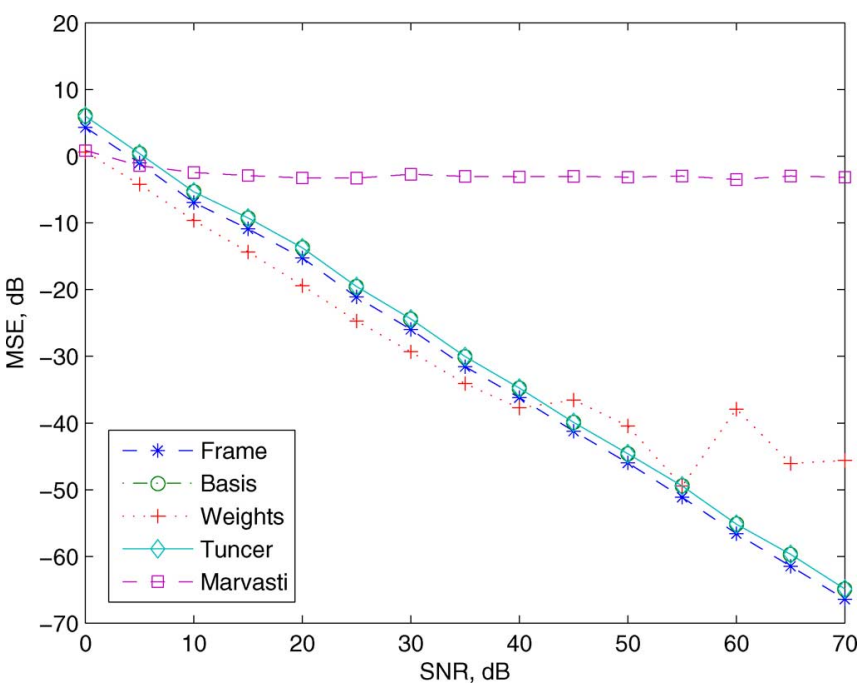

Fig. 11. Reconstruction of periodic 25 bandlimited signals from $N=65$ nonuniform samples.

where $\tau_{p}$ is a random integer uniformly distributed in $[0, L)$ and its value is known. This method interpolates the uniform samples $x\left(t_{u}\right)$ from the set of nonuniform samples $x\left(t_{p}\right)$ by solving the system

$$
\frac{1}{L N^{2}} \mathbf{W}_{1}^{H} \mathbf{H C}_{3} \mathbf{W}_{2} x\left(t_{u}\right)=\mathbf{C}_{2} x\left(t_{p}\right)
$$

where $\mathbf{H}$ is a $L N \times L N$ circulant matrix derived from the DFT of the sampling function, $\mathbf{C}_{2}$ and $\mathbf{C}_{3}$ are $L N \times N$ expansion matrices, and $\mathbf{W}_{1}$ and $\mathbf{W}_{2}$ are $L N \times L N$ and $N \times N$ DFT matrices, respectively.

In our experiment, we considered a 10-periodic 25-bandlimited signal. We chose $N=65$ nonuniform samples taken according to the nonuniform grid defined in (76) with $L=10$. This set of samples is corrupted by white Gaussian noise, which is normalized to fit the signal-to-noise ratio (SNR) value of the current simulation. The oversampling ratio of this sampling set is $r=1.27$. We performed 100 trials per SNR value and the average result is presented. The periodic bandlimited signal and the noise sequence are randomly generated for each trial.

Fig. 11 shows the MSE performance of different algorithms. The MSE of Marvasti's algorithm is very large. We can clearly see from Fig. 11 that Tuncer's results coincide with our method presented in Theorem 1. Evidently, the frame-based reconstruction provides better results compared to the reconstruction with a basis. We also observe that the adaptive weights method shows good behavior, but for SNR $>40 \mathrm{~dB}$, our frame-based approach provides the lowest reconstruction error.

\section{Comparison of the Reconstruction Methods Without Noise}

We now compare the performance of the proposed algorithms in the ideal condition, where the noise is equal to zero. The periodic bandlimited signal is generated, sampled, and reconstructed similarly to the previous subsection with $N=65$ and $L=10$. We provide simulations for different values of $K$, from $K=32$ to $K=16$, for which the oversampling ratios vary from $\kappa=1$ to $\kappa=1.97$. 


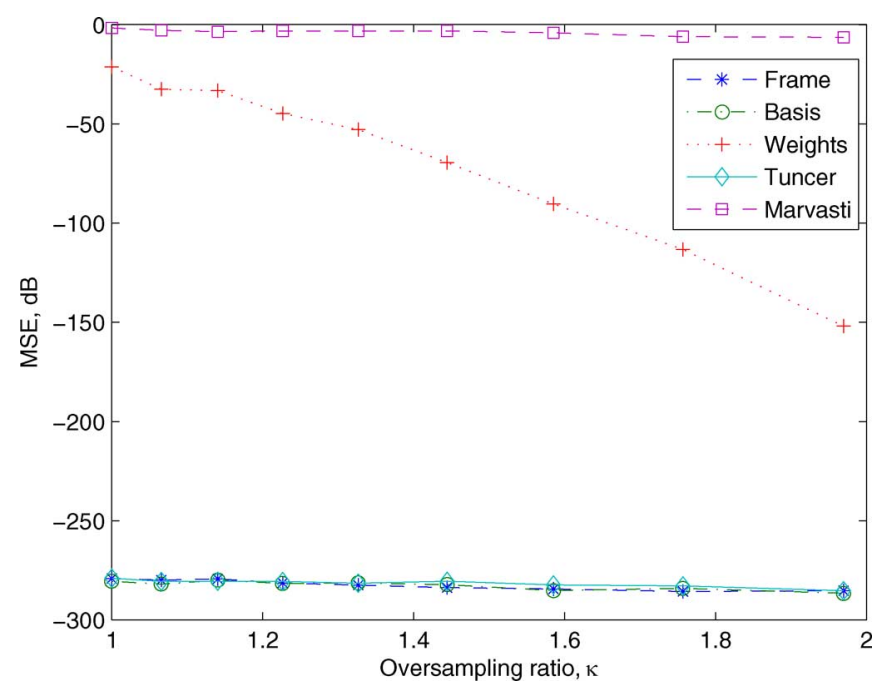

Fig. 12. Reconstruction of periodic 25 bandlimited signals from $N=65$ nonuniform samples.

The MSE values of the reconstruction as a function of $\kappa$ are presented in Fig. 12. For the methods of Theorems 1 and 3 and Tuncer's algorithm, we expect perfect reconstruction (i.e., $\mathrm{MSE}=-\infty)$. However, we see that due to the finite precision of the computations, very small reconstruction errors are present. We can see that Marvasti's and the adaptive weights methods do not converge to the original signal. We also observe that the reconstruction quality of the adaptive weights algorithm depends on the oversampling ratio. Evidently, the behavior for signals with an average sampling rate equal to the Nyquist rate (i.e., $N=2 K+1$ ) is poor.

\section{Reconstruction of Nonperiodic Bandlimited Signals}

We now focus on the reconstruction of nonperiodic bandlimited signals from nonuniform samples. For this purpose, we use the overlap-save method described in [38], where the sampled signal is divided into overlapping blocks. Each block is then assumed to be a periodic bandlimited signal and reconstructed using one of the methods presented in this paper. To minimize the boundary effects of this approach, $Q$ samples from the beginning and end of each reconstructed block are discarded. Therefore, two consequent blocks overlap by $2 Q$ samples.

In this experiment, we generated a random nonperiodic signal and filtered it using a Kaiser filter with high stopband attenuation. Then, 1024 nonuniform samples are chosen according to (76) with $L=10$ corresponding to a redundancy ratio of $r=1.05$. The samples are corrupted by white Gaussian noise, normalized to fit the SNR value of the current simulation. The signal is then reconstructed from the set of noisy samples by using the overlap-save method with $N=64$ samples in each block and $Q=5$. As in the previous examples, we perform 100 simulations per SNR value and present the average result.

The results are presented in Fig. 13. In the case of nonperiodic bandlimited signals, Marvasti's method shows better performance compared to the reconstruction of periodic signals. In contrast, the performance of the adaptive weights method is worse compared to the results of Fig. 11. This behavior can be

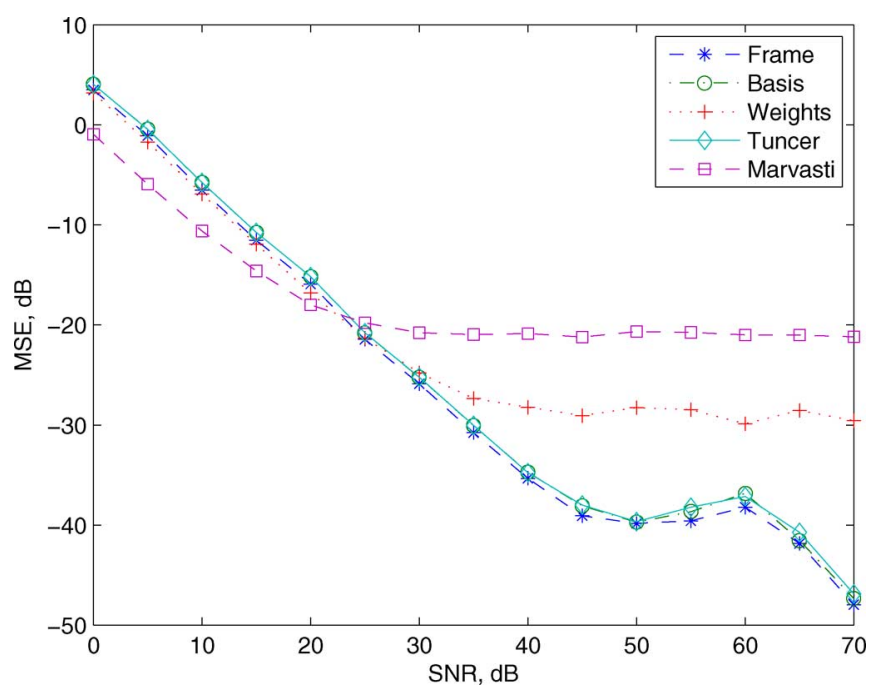

Fig. 13. Reconstruction of nonperiodic bandlimited signals from 1024 nonuniform samples using the overlap-save method. Each overlapped block has $N=$ 64 nonuniform samples.

explained by noting that $\kappa=1.05$ in this example. We also observe that similar to Fig. 11, reconstruction with basis and Tuncer's methods are equivalent, and the frame-based reconstruction performs better than these two methods.

We summarize this section by noting that the two reconstruction methods derived in this paper have solutions for an arbitrary distribution of nonuniform samples that satisfies $N \geq 2 K+1$. Both algorithms provide closed-form solutions without complex-valued arithmetic. The frame-based reconstruction of Theorem 3 typically provides a better quality of reconstruction, exploiting redundancy of the sampling set. Finally, in the ideal case, perfect reconstruction is guaranteed by both methods.

\section{CONCLUSION}

In this paper, we developed two new formulas for reconstruction of periodic bandlimited signals from nonuniformly spaced samples. The first formula provides new reconstruction functions for the case of an even number of sampling points. The second reconstruction formula was developed for the case of oversampled periodic bandlimited signals. We compared the two proposed methods and examined the advantages and drawbacks of each. Specifically, we showed that the first method provides consistent reconstruction of the signal while the second is more stable in noisy environments. In order to characterize the relation between the discrete-time noise of the samples and the continuous-time error of the reconstruction, we have used the condition number. The exact formulas for calculation of the condition number were derived. These conclusions were supported by theoretical derivations and simulation results.

Finally, we investigated an efficient implementation of the reconstruction methods for uniform and recurrent nonuniform sampling. In the case of uniform samples, we calculated the precise value of the condition number and developed a simple formula for calculation of the condition number in the case of recurrent nonuniform sampling. The performance of our methods compared to other algorithms was demonstrated with the reconstruction of periodic and nonperiodic bandlimited signals. 


\section{APPENDIX A \\ FOURIER COEFFICIENTS CALCULATION}

Using the exponential decomposition of sine functions, we can express the product of sines in (23) as a sum of exponents

$$
\begin{aligned}
& \prod_{\substack{q=0 \\
q \neq p}}^{N-1} \sin \left(\frac{\pi\left(u+t_{p}-t_{q}\right)}{T}\right) \\
& =\prod_{\substack{q=0 \\
q \neq p}}^{N-1} \frac{1}{2 j}\left(e^{j \pi\left(u+t_{p}-t_{q}\right) / T}-e^{-j \pi\left(u+t_{p}-t_{q}\right) / T}\right) \\
& =\sum_{\substack{l=-N+1 \\
N-1}}^{N} b_{l} e^{j \pi l u / T}
\end{aligned}
$$

where the complex coefficients $b_{l}$ are the result of expanding the product of sines. The power of each exponent in (79) is obtained as a sum of $m$ positive and $N-1-m$ negative powers of exponents in (78). Therefore, the set of all possible values of $l$ is defined as $l=m-(N-1-m)=2 m-N+1$, for $m \in[0, N-1]$. As a result

$$
b_{l}=0, \quad l=-N+2,-N+4, \ldots, N-2 .
$$

Since the coefficients $b_{l}$ will be used to represent the final expression as the product of sines, the exact values of the coefficients are not needed.

We observe that the intervals $I_{n}$ in (23) are not overlapping and the union of all intervals $I_{n}$ is the set of all real numbers (i.e., $\bigcup_{n=-\infty}^{\infty} I_{n}=\mathbf{R}$ ). For this reason, we replace the sum and integral operations in (23) by an integral over the domain $u \in[-\infty, \infty]$. The coefficients (23) then becomes

$$
\begin{aligned}
c_{k}= & a_{p} e^{-j 2 \pi k t_{p} / T} \\
& \times \frac{1}{T} \int_{-\infty}^{\infty} \frac{\sin \left(\frac{\pi u}{T}\right)}{\frac{\pi u}{T}} \sum_{l=-N+1}^{N-1} b_{l} e^{j \pi l u / T} e^{-j 2 \pi k u / T} d u \\
= & a_{p} e^{-j 2 \pi k t_{p} / T} \\
& \times \sum_{l=-N+1}^{N-1} \frac{b_{l}}{T} \int_{-\infty}^{\infty} \frac{\sin \left(\frac{\pi u}{T}\right)}{\frac{\pi u}{T}} e^{-j(2 \pi / T)(k-l / 2) u} d u .
\end{aligned}
$$

Using the fact that

$$
\frac{\operatorname{sinc}\left(\frac{u}{T}\right)}{T} \stackrel{\text { Fourier }}{\longleftrightarrow} \operatorname{rect}(T f)= \begin{cases}1, & |T f|<0.5 \\ 0.5, & |T f|=0.5 \\ 0, & \text { otherwise }\end{cases}
$$

we obtain

$$
\begin{aligned}
c_{k} & =a_{p} e^{-j 2 \pi k t_{p} / T} \sum_{l=-N+1}^{N-1} b_{l} \operatorname{rect}\left(k-\frac{l}{2}\right) \\
& = \begin{cases}a_{p} \frac{b_{2 k-1}+b_{2 k+1}}{2} e^{-j 2 \pi k t_{p} / T}, & |k| \leq \frac{N}{2} \\
0, & \text { otherwise. }\end{cases}
\end{aligned}
$$

\section{APPENDIX B \\ TRIGONOMETRIC IDENTITY OF THEOREM 2}

Using the trigonometric identities

$$
\begin{aligned}
& \sin (\alpha+\beta)=\sin \alpha \cos \beta+\cos \alpha \sin \beta \\
& \cos (\alpha+\beta)=\cos \alpha \cos \beta-\sin \alpha \sin \beta
\end{aligned}
$$

the numerator of (36) for a given value $p$ can be written as

$$
\begin{aligned}
& \frac{\cos \left(\sum_{q=0, q \neq p, q \neq i}^{N-1}\left(\frac{\pi\left(t_{p}-t_{q}\right)}{T}\right)\right)}{\prod_{q=0, q \neq p, q \neq i}^{N-1} \sin \left(\frac{\pi\left(t_{p}-t_{q}\right)}{T}\right)} \\
& \quad+\cot \left(\frac{\pi\left(t_{p}-t_{i}\right)}{T}\right) \frac{\sin \left(\sum_{q=0, q \neq p, q \neq i}^{N-1}\left(\frac{\pi\left(t_{p}-t_{q}\right)}{T}\right)\right)}{\prod_{q=0, q \neq p, q \neq i}^{N-1} \sin \left(\frac{\pi\left(t_{p}-t_{q}\right)}{T}\right)} .
\end{aligned}
$$

Applying (84) recursively onto (85) and summating over $p$, converts (36) into an expression consisting of the products of an even number of cotangent functions, which are summed over all possible combinations of its arguments (i.e., over all possible combinations of $\left(t_{p}-t_{q}\right)$, for $\left.p \neq q\right)$. The number of the combinations of the products of $n$ (even) cotangents is $N\left(\begin{array}{c}N-1 \\ n\end{array}\right)$. Combining these products into the groups of $m=n+1$ sums, allows the use of the following equation, which is taken from the standard tables of finite trigonometric series [39, p. 647]

$$
\sum_{p=1}^{m} \prod_{\substack{q=1 \\ q \neq p}}^{m} \cot \left(\frac{\pi\left(t_{p}-t_{q}\right)}{T}\right)=\sin \left(\frac{m \pi}{2}\right) .
$$

For $n$ even ( $m$ odd), (86) is reduced to

$$
\sum_{p=1}^{m} \prod_{\substack{q=1 \\ q \neq p}}^{m} \cot \left(\frac{\pi\left(t_{p}-t_{q}\right)}{T}\right)=(-1)^{(m-1) / 2} .
$$

Integrating the products of cotangents of (85) into such groups and using (87), we can evaluate the expression (85) as

$$
\begin{aligned}
(-1)^{(N / 2-1)} & \sum_{k=0}^{N / 2-1} N\left(\begin{array}{c}
N-1 \\
2 k
\end{array}\right) \frac{1}{2 k+1} \\
= & (-1)^{(N / 2-1)} \sum_{k=0}^{N / 2-1} \frac{N(N-1) !}{(N-1-2 k) !(2 k) !(2 k+1)} \\
& =(-1)^{(N / 2-1)} \sum_{k=0}^{N / 2-1} \frac{N !}{(N-(2 k+1)) !(2 k+1) !} \\
& =(-1)^{(N / 2-1)} \sum_{k=0}^{N / 2-1}\left(\begin{array}{c}
N \\
2 k+1
\end{array}\right) \\
& =(-1)^{(N / 2-1)} 2^{N-1}
\end{aligned}
$$

where the last equation results directly from [39, p. 607]. 


\section{APPENDIX C \\ PRODUCT OF SINES FOR UNIFORM SAMPLES}

To simplify the expression for $h_{p}(t)$ for uniform samples, we first introduce two trigonometric identities [39, p. 752]

$$
\prod_{k=0}^{N-1} \sin \left(\theta+\frac{k \pi}{N}\right)=\frac{\sin (N \theta)}{2^{N-1}}
$$

and $[39$, p. 752]

$$
\prod_{k=1}^{N-1} \sin \frac{k \pi}{N}=\frac{N}{2^{N-1}} .
$$

Substituting the set of uniform samples $t_{p}=p T / N$ into the product of sine functions of (15), we have

$$
\begin{aligned}
& \prod_{\substack{q=0 \\
q \neq p}}^{N-1} \frac{\sin \left(\frac{\pi\left(t-t_{q}\right)}{T}\right)}{\sin \left(\frac{\pi\left(t_{p}-t_{q}\right)}{T}\right)} \\
& =\frac{\prod_{q=0}^{N-1} \sin \left(\frac{\pi\left(t-t_{q}\right)}{T}\right)}{\sin \left(\frac{\pi\left(t-t_{p}\right)}{T}\right) \prod_{\substack{q=0 \\
q \neq p}}^{N-1} \sin \left(\frac{\pi\left(t_{p}-t_{q}\right)}{T}\right)} \\
& =\frac{\prod_{q=0}^{N-1} \sin \left(\frac{\pi}{T} t-\frac{q \pi}{N}\right)}{\sin \left(\frac{\pi\left(t-t_{p}\right)}{T}\right) \prod_{\substack{q=0 \\
q \neq 0}}^{N-1} \sin \left(\frac{(p-q) \pi}{N}\right)} \\
& =\frac{(-1)^{N-1} \prod_{q=0}^{N-1} \sin \left(\frac{\pi}{T} t+\frac{q \pi}{N}\right)}{\sin \left(\frac{\pi\left(t-t_{p}\right)}{T}\right)(-1)^{N-1} \prod_{k=1}^{N-1} \sin \left(\frac{k \pi}{N}\right)}
\end{aligned}
$$

where the last equation results from the fact that $\sin (\theta)=$ $-\sin (-\theta)$ and $\sin (\theta-\varphi \pi)=-\sin (\theta+(1-\varphi) \pi)$. Using (89) and (90), we develop an equivalent representation for (91) as

$$
\frac{2^{N-1} \sin \left(\frac{N \pi t}{T}\right)}{N 2^{N-1} \sin \left(\frac{\pi\left(t-t_{p}\right)}{T}\right)}=\frac{\sin \left(\frac{N \pi\left(t-t_{p}\right)}{T}\right)}{N \sin \left(\frac{\pi\left(t-t_{p}\right)}{T}\right)}
$$

completing the proof.

\section{APPENDIX D}

\section{EXPONENTIAL EXPANSIONS FOR UNIFORM SAMPLES}

We now express the functions of (44) in exponential form, where for simplicity, $t-t_{p}$ is replaced by $\tau$. For $N$ odd

$$
h_{p}(t)=\frac{1}{N} \frac{e^{j \pi N \tau / T}-e^{-j \pi N \tau / T}}{e^{j \pi \tau / T}-e^{-j \pi \tau / T}}
$$

and for $N$ even

$$
h_{p}(t)=\frac{\left(e^{j \pi \tau / T}+e^{-j \pi \tau / T}\right)\left(e^{j \pi N \tau / T}-e^{-j \pi N \tau / T}\right)}{2 N\left(e^{j \pi \tau / T}-e^{-j \pi \tau / T}\right)} .
$$

Applying the identity

$$
x^{N}-x^{-N}=\left(x-x^{-1}\right)\left(x^{N-1}+x^{N-3}+\cdots+x^{-(N-1)}\right)
$$

on the term $\left(e^{j \pi N \tau / T}-e^{-j \pi N \tau / T}\right)$ of (93) and (94), results in the reduction of denominators in both expressions. Orthogonal projection of these functions onto the space $V_{K}$ results in

$$
h_{p}(t)=\frac{1}{N} \sum_{l=-K}^{K} e^{j 2 \pi l \tau / T}
$$

for both $N$, even and odd. Using similar derivations, the last function (96) can be expressed in trigonometric form (44).

\section{REFERENCES}

[1] A. Cauchy, "Mémoire sur diverses formules d'analyse," C. R. Acad. Sci., vol. 12, pp. 283-298, 1841.

[2] C. E. Shannon, "Communication in the presence of noise," Proc. IRE, vol. 37, pp. 10-21, Jan. 1949.

[3] R. J. Marks, II, "Restoring lost samples from an oversampled bandlimited signal," IEEE Trans. Acoust., Speech, Signal Process., vol. ASSP-31, no. 3, pp. 752-755, Jun. 1983.

[4] P. J. S. G. Ferreira, "The stability of a procedure for the recovery of lost samples in band-limited signals," Signal Process., vol. 40, no. 3, pp. 195-205, Dec. 1994.

[5] H. Stark, "Polar, spiral and generalized sampling and interpolation," in Advanced Topics in Shannon Sampling and Interpolation Theory, R. J. Marks, II, Ed. New York: Springer-Verlag, 1993, pp. 185-218.

[6] N. Sayiner, H. V. Sorensen, and T. R. Viswanathan, "A level-crossing sampling scheme for A/D conversion," IEEE Trans. Circuits Syst. II, vol. 43, no. 4, pp. 335-339, Apr. 1996.

[7] K. Yao and J. B. Thomas, "On some stability and interpolatory properties of nonuniform sampling expansions," IEEE Trans. Circuit Theory, vol. CT-14, no. 4, pp. 404-408, Dec. 1967.

[8] J. L. Yen, "On nonuniform sampling of bandwidth-limited signals," IEEE Trans. Circuit Theory, vol. CT-3, no. 4, pp. 251-257, Dec. 1956.

[9] A. J. Jerri, "The Shannon sampling theorem-Its various extensions and applications: A tutorial review," Proc. IEEE, vol. 65, no. 11, pp. 1565-1596, Nov. 1977.

[10] Y. C. Eldar and A. V. Oppenheim, "Filterbank reconstruction of bandlimited signals from nonuniform and generalized samples," IEEE Trans. Signal Process., vol. 48, no. 10, pp. 2864-2875, Oct. 2000.

[11] R. S. Prendergast, B. C. Levy, and P. J. Hurst, "Reconstruction of bandlimited periodic nonuniformly sampled signals through multirate filter banks," IEEE Trans. Circuits Syst. I, vol. 51, no. 8, pp. 1612-1622, Aug. 2004.

[12] H. Johansson, P. Löwenborg, and K. Vengattaramane, "Least-squares and minimax design of polynomial impulse response FIR filters for reconstruction of two-periodic nonuniformly sampled signals," IEEE Trans. Circuits Syst. I, vol. 54, no. 4, pp. 877-888, Apr. 2007.

[13] T. Strohmer, "Computationally attractive reconstruction of bandlimited images from irregular samples," IEEE Trans. Image Process., vol. 6, no. 4, pp. 540-548, Apr. 1997.

[14] E. Margolis and Y. C. Eldar, "Filterbank reconstruction of periodic signals and sampling in polar coordinates," presented at the Workshop on Sampling Theory and Applications, Strobl, Austria, May 2003.

[15] F. Marvasti, M. Analoui, and M. Gamshadzahi, "Revovery of signals from nonuniform samples using iterative methods," IEEE Trans. Signal Process., vol. 39, no. 4, pp. 872-878, Apr. 1991.

[16] G. H. Feichtinger, K. Gröchenig, and T. Strohmer, "Efficient numerical methods in nonuniform sampling theory," Numer. Math., vol. 69, pp. 423-440, 1995.

[17] T. E. Tuncer, "Block-based methods for the reconstruction of finitelength signals from nonuniform samples," IEEE Trans. Signal Process., vol. 55, no. 1, pp. 530-541, Jan. 2007.

[18] A. Zygmund, Trigonometric Series, 2nd ed. Cambridge, U.K.: Cambridge Univ. Press, 1959.

[19] J. L. Brown Jr., "Cauchy and polar-sampling theorems," J. Opt. Soc. Am. A, vol. 1, pp. 1054-1056, Oct. 1984.

[20] F. A. Marvasti, A Unified Approach to Zero-Crossings and Nonuniform Sampling. Chicago, IL: Illinois Inst. Technol., 1987.

[21] P. J. S. G. Ferreira, "Interpolation and the discrete Papoulis-Gerchberg algorithm," IEEE Trans. Signal Process., vol. 42, no. 10, pp. 2596-2606, Oct. 1994.

[22] P. P. Vaidyanathan and V. C. Liu, "Efficient reconstruction of bandlimited sequences from nonuniformly decimated versions by use of polyphase filter banks," IEEE Trans. Acoust., Speech, Signal Process., vol. 38, no. 11, pp. 1927-1936, Nov. 1990. 
[23] R. J. Duffin and A. C. Schaeffer, "A class of nonharmonic Fourier series," Trans. Amer. Math. Soc., vol. 72, pp. 341-366, 1952.

[24] O. Christensen, An Introduction to Frames and Riesz Bases. Cambridge, MA: Birkhäuser, 2002.

[25] Y. C. Eldar, "Sampling without input constraints: Consistent reconstruction in arbitrary spaces," in Sampling, Wavelets and Tomography, A. Zayed and J. J. Benedetto, Eds. Cambridge, MA: Birkhäuser, 2003.

[26] M. Unser and J. Zerubia, "Generalized sampling: Stability and performance analysis," IEEE Trans. Signal Process., vol. 45, no. 12, pp. 2941-2950, Dec. 1997.

[27] H. Stark, "Sampling theorems in polar coordinates," J. Opt. Soc. Amer., vol. 69, pp. 1519-1525, Nov. 1979.

[28] H. Stark and M. Wengrovitz, "Comments and corrections on the use of sampling theorems in CT," IEEE Trans. Acoust., Speech, Signal Process., vol. ASSP-31, no. 5, pp. 1329-1331, Oct. 1983.

[29] T. Schanze, "Sinc interpolation of discret periodic signals," IEEE Trans. Signal Process., vol. 43, no. 6, pp. 1502-1503, Jun. 1995.

[30] J. L. Brown Jr., "An RKHS analysis of sampling theorems for harmonic-limited signals," IEEE Trans. Acoust., Speech, Signal Process., vol. ASSP-33, no. 2, pp. 437-440, Apr. 1985.

[31] E. Margolis, "Reconstruction of periodic bandlimited signals from nonuniform samples," M.S. degree, Technion-Israel Inst. Technol., Haifa, Israel, 2004.

[32] S. Mallat, A Wavelet Tour of Signal Processing. London, U.K.: Academic Press, 1999.

[33] R. M. Gray, "Quantization noise spectra," IEEE Trans. Inf. Theory, vol. 36, no. 1, pp. 1220-1240, Jan. 1990.

[34] V. K. Goyal, M. Vetterli, and N. T. Thao, "Quantized overcomplete expansions in $\mathbb{R}^{n}$ : Analysis, synthesis, and algorithms," IEEE Trans. Inf. Theory, vol. 44, no. 1, pp. 16-31, Jan. 1998.

[35] Y. C. Eldar, "Sampling with arbitrary sampling and reconstruction spaces and oblique dual frame vectors," J. Fourier Anal. Appl., vol. 9, no. 1, pp. 77-96, Jan. 2003.

[36] R. M. Gray, "Toeplitz and circulant matrices: A review," Tech. Rep. Standford, CA, 2002, Dept. Elect. Eng., Inf. Syst. Lab., Stanford Univ.

[37] A. Beck, Y. C. Eldar, and A. Ben-Tal, "Mean-squared error estimation for linear systems with block circulant uncertainty," SIAM J. Matrix Anal. Appl., vol. 29, no. 3, pp. 712-730, Jun. 2007.

[38] A. V. Oppenheim, R. W. Schafer, and J. R. Buck, Discrete-Time Signal Processing, 2nd ed. Upper Saddle River, NJ: Prentice-Hall, 1999.

[39] A. P. Prudnikov, Y. A. Brychkov, and O. I. Marichev, Integral and Series. New York: Gordon and Breach, 1986, vol. 1, Elementary Functions.

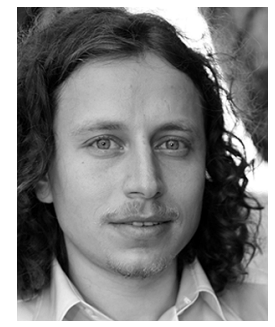

Evgeny Margolis (S'04) received the B.Sc. and M.Sc. degrees in electrical engineering from the Technion-Israel Institute of Technology, Haifa, Israel, in 2001 and 2004, respectively.

From 1998 to 2001, he was with the Intel Corporation, Haifa, Israel. From 2001 to 2004, he was a Research Assistant and a Project Supervisor with the Signal and Image Processing Lab (SIPL), Faculty of Electrical Engineering, Technion. Since 2004, he has been a System Architect with the Wireless Subscriber Group, Freescale Semiconductor, Chandler, AZ. His main areas of interests include digital communication algorithms, signal-processing algorithms, and sampling theory with applications in image processing and communication.

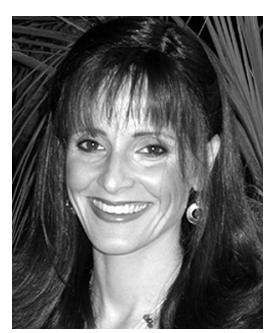

Yonina C. Eldar (S'98-M'02-SM'07) received the B.Sc. degree in physics and the B.Sc. degree in electrical engineering from Tel-Aviv University (TAU), Tel-Aviv, Israel, in 1995 and 1996, respectively, and the Ph.D. degree in electrical engineering and computer science from the Massachusetts Institute of Technology (MIT), Cambridge, in 2001.

In 2002, she was a Postdoctoral Fellow at the Digital Signal Processing Group at MIT. Currently, she is an Associate Professor in the Department of Electrical Engineering at the Technion-Israel Institute of Technology, Haifa, Israel. She is also a Research Affiliate with the Research Laboratory of Electronics at MIT. Her research interests are in the general areas of signal processing, statistical signal processing, and computational biology.

Dr. Eldar was in the program for outstanding students at TAU from 1992 to 1996. In 1998, she held the Rosenblith Fellowship for study in Electrical Engineering at MIT and, in 2000, she held an IBM Research Fellowship. From 2002 to 2005, she was a Horev Fellow of the Leaders in Science and Technology program at the Technion and an Alon Fellow. In 2004, she was awarded the Wolf Foundation Krill Prize for Excellence in Scientific Research, the Andre and Bella Meyer Lectureship in 2005, the Henry Taub Prize for Excellence in Research in 2007, and the Hershel Rich Innovation Award and Award for Women with Distinguished Contributions in 2008. She is a member of the IEEE Signal Processing Theory and Methods Technical Committee, an Associate Editor with the IEEE TRANSACTIONS ON Signal PROCESSING, the EURASIP Journal of Signal Processing, and the SIAM Journal on Matrix Analysis and Applications, and on the Editorial Board of Foundations and Trends in Signal Processing. 